\title{
Experimental validation of piezoelectric shunt tuning with residual mode correction: Damping of plate-like structures
}

\author{
Toftekær, Johan Frederik; Høgsberg, Jan Becker
}

Published in:

Journal of Intelligent Material Systems and Structures

Link to article, DOI:

10.1177/1045389X20914393

Publication date:

2020

Document Version

Peer reviewed version

Link back to DTU Orbit

Citation (APA):

Toftekær, J. F., \& Høgsberg, J. B. (2020). Experimental validation of piezoelectric shunt tuning with residual mode correction: Damping of plate-like structures. Journal of Intelligent Material Systems and Structures, 31(9), 220-1239. https://doi.org/10.1177/1045389X20914393

\section{General rights}

Copyright and moral rights for the publications made accessible in the public portal are retained by the authors and/or other copyright owners and it is a condition of accessing publications that users recognise and abide by the legal requirements associated with these rights.

- Users may download and print one copy of any publication from the public portal for the purpose of private study or research.

- You may not further distribute the material or use it for any profit-making activity or commercial gain

- You may freely distribute the URL identifying the publication in the public portal 


\title{
EXPERIMENTAL VALIDATION OF PIEZOELECTRIC SHUNT TUNING WITH RESIDUAL MODE CORRECTION: DAMPING OF PLATE-LIKE STRUCTURES
}

\author{
JOHAN FREDERIK TOFTEKÆR AND JAN HØGSBERG \\ DEPARTMENT OF MECHANICAL ENGINEERING \\ TECHNICAL UNIVERSITY OF DENMARK \\ NILS KOPPELS ALLÉ, BUILDING 404, DK-2800 KGS. LYNGBY, DENMARK
}

\begin{abstract}
Excessive resonant vibration of flexible plate-like structures may be mitigated by the attachment of co-located piezoceramic patches wired to a passive resonant shunt realized by a passive inductor, composed of a cobber wire around a magnetic coil and a simple resistor. While the pure passive shunt damping guarantees unconditional stability it seemingly requires an exact prior inductance tuning for determining the exact number of wire winds. Traditional shunt tuning methods based on the blocked or static capacitance, from a single mode representation, may therefore be insufficient. A recently proposed shunt tuning method includes the residual modes contribution by the evaluation of an effective modal capacitance based on the modal charge and voltage associated with the short- and open circuited (SC and $\mathrm{OC}$ ) piezoelectric absorber. An experimental implementation of this shunt tuning method is proposed and validated by experiments with a free beam and plate with five pairs of piezoceramic patches. The experimental method consists of imposing pseudo-random vibrations to the analyzed structure, while the piezoelectric current and voltage response are measured for the SC and OC piezoelectric absorbers, respectively. Subsequently, the corresponding frequency response functions (FRF) are determined for the SC charge determined from the measured current and the OC voltage. Finally, the modal charge and voltage amplitudes are found as the peak values in their respective FRFs, and then used to determine the effective modal capacitance. Good correlation between the numerical and experimental shunt tuning methods are demonstrated for the tuning and damping of four target modes of the free beam, while optimum multi-mode shunt tuning is obtained by the determination of a modified effective electromechanical coupling coefficient, as demonstrated for simultaneous damping of two and four free plate modes.
\end{abstract}

\section{INTRODUCTION}

Flexible plate-like structures may be vulnerable to excessive vibrations caused by dynamic harmonic loads with frequencies close to a resonance in the structure. This may cause structural failure, fatigue or lead to undesirable acoustic problems. The introduction of resonant absorber devices calibrated to a single or several dominating resonant modes of the structure may therefore be required. Regarding vibration mitigation of plate-like structures, a convenient absorber device consists of co-located piezoceramic patches with strong electromechanical coupling properties wired to resonant shunts. The resonant shunt consists of an inductance $(L)$ tuned to secure a proper absorber frequency and a resistor $(R)$ chosen to obtain optimum energy dissipation around the targeted resonant frequency, as proposed and experimentally demonstrated by [6]. A tuning procedure based on a single mode approximation of the electromechanical structure has been derived subsequently for the series [8] and parallel [24] $L R$-shunts, while a comparison of the two shunt configurations have been provided by [16] and [3]. Alternative methods for the optimum series and parallel resonant shunt tuning has been suggested in [20, 26, 9, 19], all relying on a pure single-mode truncation. A consequence of assuming the single mode approach is that the optimum inductance tuning is evaluated directly from the inherent blocked piezoelectric capacitance $C_{p}^{S}$ in order to obtain the desired absorber frequency. However, due to the effect of non-resonant vibration modes this will in many cases lead to a non-optimum inductance and shunt tuning.

Key words and phrases. Piezoelectric shunt damping, resonant shunt calibration, residual mode correction, effective electromechanical coupling coefficient. 
The effect of the non-resonant vibration modes has rarely been encountered in both numerical and experimental validations of shunt tuning procedures presented in the literature. In the case of numerical validations, this is often because a modal truncation is used when producing the frequency response of the electromechanical structure [3, 19], whereby the effect from nonresonant vibration modes are naturally avoided, while in experimental validations it is rarely stated precisely which piezoelectric capacitance is measured and used in the shunt tuning. The difference between the blocked piezoelectric capacitance and the capacitance of a piezoelectric element bonded to a vibrating structure has been clarified a few times in the literature. In [25] it is stated that the capacitance of a piezoceramic patch bonded to a structure is only slightly different than the inherent blocked capacitance, which is therefore kept as the governing shunt tuning parameter. In $[17,15,18]$ the effective capacitance of a piezoelectric element bonded to a vibrating structure is discussed in greater detail. [17] suggests four different capacitances, the original free and blocked capacitances and then two additional capacitances: The free modal and the blocked modal capacitance of the piezoelectric element bonded to a vibrating structure. The free modal capacitance can be measured when the structure is free to deform and it may therefore be measured at very low frequency, hence in $[15,18]$ it is denoted the static capacitance $C_{p}^{0}$. The blocked modal capacitance is obtained when all modal coefficients are blocked, which is more difficult to determine directly from experimental measurement. However, as described in [17] the optimum shunt tuning should be based on this blocked modal capacitance since it also contains contributions from the non-resonant vibration modes. Finally, two method for the evaluation of the blocked modal capacitance is proposed, one that requires the use of a synthetic inductor, and an other that is associated with significant uncertainties because the effective electromechanical coupling coefficient (EMCC) must be determined precisely.

Recently, the influence of the non-resonant vibration modes on the optimum resonant shunt tuning has been further considered. In [10] explicit flexibility and inertia correction terms, initially derived for tuned mass- and inerter based absorbers in [12], have been proposed. In [1, 2] the inherent capacitance of the structure bonded piezoelectric element is modified by the influence from higher vibration modes, while a theoretical study of the influence from an increasing number of vibration modes on the shunt tuning has been provided in [7]. An electrical analogy to the flexibility and inertia correction terms in [10] has been proposed in [21], where it is demonstrated that the corresponding electric capacitance and inductance correction terms, together with the inherent blocked piezoelectric capacitance, determines an effective dynamic capacitance used to evaluate and improve the traditional shunt inductance tuning. In [22] the effect of distinguishing between the flexibility and inertia corrections from the non-resonant vibration modes is found to have insignificant influence on the final practical shunt performance. Therefore, an alternative effective capacitance based on the electric response to a short and a open circuit (SC and OC) eigenvalue problem is proposed in [23]. This method is simple to implement in commercial finite element (FE) software [22] as it only requires the evaluation of two eigenvalue problems and thus avoids any matrix manipulations or determination of an extensive number of non-resonant frequencies and mode shapes. The method is further suitable for experimentally based shunt calibration, as demonstrated in the present paper.

The present paper aims to provide an experimental validation of the shunt tuning procedure proposed in [23] and to experimentally demonstrate the effect from non-resonant vibration modes on the optimum shunt calibration. The latter is done by considering the optimum shunt tuning based on both the blocked, the static and the effective modal capacitance [23]. The shunt circuits are designed as pure passive electrical circuits with use of a passive inductor, designed by winding a cobber wire around a closed magnetic coil [14], connected in series with a potentiometers, which is easily adjusted to the specific resistance tuning. As it will be discussed through out the paper, the use of the passive inductor increases the importance of a precise prior inductance tuning, since subsequent re-adjustment of the final passive inductance is not as straight forward as for the synthetic inductor. Finally, the multi-mode piezoelectric shunt tuning proposed in [23] is demonstrated experimentally.

The paper is organized in three main sections. In the first section, the numerical shunt tuning method proposed in [23] is briefly summarized for the series shunt, used in the experiments. 
Furthermore, the numerical results for the initial experimental setup, concerning a free beam with 5 piezoceramic patch pairs, is presented for subsequent comparison with the corresponding experiments. In the second section, it is demonstrated how the required shunt tuning parameters can be evaluated experimentally by measurements of the piezoelectric SC current and OC voltage absorber response to a specific dynamic load. The passive inductor is then briefly presented, which requires a precise prior tuning that includes the influence from the residual modes. In the third section, two experimental setups concerning a free beam and plate, each with 5 pairs of piezoceramic patches, are presented and the optimum shunt tuning to four of the piezoceramic patch pairs is determined from the experimentally evaluated shunt tuning parameters. Finally, the shunt damping performance is verified by considering the frequency response functions (FRF) for the structural displacements and the piezoelectric absorber voltage response around the targeted resonance vibration modes.

\section{NUMERICAL SHUNT TUNING}

This section presents the numerical model and shunt tuning method [23] to be validated experimentally in the subsequent sections. In a frequency domain representation with angular frequency $\omega$, the general vibration problem for an elastic structure with attached piezoelectric domains may be described as [22]

$$
\left(\left[\begin{array}{cc}
K^{E} & k_{m e}^{E} \\
\left(k_{m e}^{E}\right)^{t} & -C_{p}
\end{array}\right]-\omega^{2}\left[\begin{array}{cc}
M & 0 \\
0 & 0
\end{array}\right]\right)\left\{\begin{array}{l}
U \\
V
\end{array}\right\}=\left\{\begin{array}{c}
0 \\
-Q
\end{array}\right\}
$$

in which $\left[K^{E}\right]$ is the elastic stiffness matrix of the structure with SC piezoelectric electrodes and $[M]$ is the mass matrix. The coupling between the mechanical and the electrical domains is governed by the coupling matrix $\left[k_{m e}^{E}\right]$ with rows equal to the number of mechanical degrees of freedom (dofs) and columns equal to the number of independent piezoelectric shunts $N_{p}$, while $(\ldots)^{t}$ represents the transpose operation. A piezoelectric shunt $Z_{i}$ may be wired to several piezoelectric domains whereby the shunted domains act as a single resulting piezoelectric absorber, see Figure 1. The electrical domain is hereby described by a diagonal matrix $\left[C_{p}\right]$ containing the resulting blocked capacitance $\left(\bar{C}_{p}^{S}\right)_{i}$ for each piezoelectric absorber [22]. In (1) the vector $\{U\}$ contains the mechanical $N$ degrees of freedom (dofs), $\{V\}$ contains the $N_{p}$ electric dofs given by the voltage between the shunted electrodes, while $\{Q\}$ represents the stored electric charge on each of the shunted electrodes, alternatively expressed by the flow of current

$$
I_{i}=-\mathrm{i} \omega Q_{i}
$$

with $\mathrm{i}=\sqrt{-1}$ being the imaginary unit.

The proposed shunt tuning method in [23] considers the two limiting eigenvalue problems from (1) with either SC and OC piezoelectric electrodes. The SC eigenvalue problem is obtained by direct wiring of the piezoelectric electrodes $\left(Z_{i}=0\right)$, whereby $\{V\}=\{0\}$ in (1) directly gives

$$
\left(\left[K^{E}\right]-\omega_{j}^{2}[M]\right)\left\{U_{j}\right\}=\{0\}
$$

introducing the SC natural frequency $\omega_{j}$ and the mode shape $\left\{U_{j}\right\}$ for mode $j$. The electric absorber response from the second equation in (1) is then obtained as a modal charge

$$
\left\{Q_{j}\right\}=-\left[k_{m e}^{E}\right]^{t}\left\{U_{j}\right\}
$$

The modal charge $\left\{Q_{j}\right\}=\left\{I_{j}\right\} /\left(\mathrm{i} \omega_{j}\right)$ is alternatively obtained from the flow of current $\left\{I_{j}\right\}$ by (2) with $\omega=\omega_{j}$, which can be readily measured in an experimental analysis. The OC limit corresponds to infinite shunt resistance $\left(Z_{i} \rightarrow \infty\right)$, implying a vanishing flow of current or $\{Q\}=$ $\{0\}$. Elimination of $\{V\}$ in the upper equation of (1) then determines the OC eigenvalue problem as

$$
\left(\left[K^{E}\right]+\left[k_{m e}^{E}\right]\left[C_{p}\right]^{-1}\left[k_{m e}^{E}\right]^{t}-\hat{\omega}_{j}^{2}[M]\right)\left\{\hat{U}_{j}\right\}=\{0\}
$$

defining the OC frequency $\hat{\omega}_{j}$ and mode shape $\left\{\hat{U}_{j}\right\}$ for a vibration mode $j$. For $\{Q\}=\{0\}$ the second equation of (1) defines the modal voltage

$$
\left\{\hat{V}_{j}\right\}=\left[C_{p}\right]^{-1}\left[k_{m e}^{E}\right]^{t}\left\{\hat{U}_{j}\right\}
$$




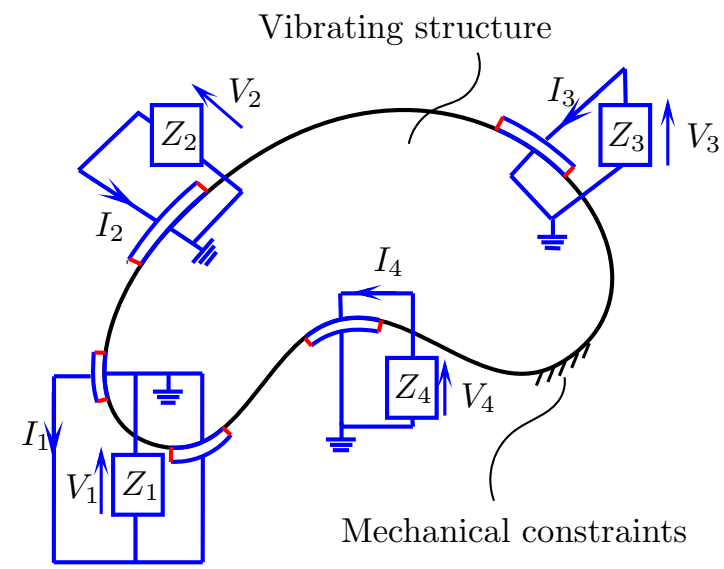

FiguRE 1. Vibrating structure with piezoelectric domains comprised by $N_{p}=4$ piezoelectric absorbers with independent shunt impedance $Z_{i}$. [23]

as the associated electric response amplitude. The evaluation of the two limiting (SC and OC) eigenvalue problems determine the SC frequencies $\omega_{j}$ and modal charge amplitudes $\left\{Q_{j}\right\}$ and the corresponding OC frequencies $\hat{\omega}_{j}$ with associated modal voltages $\left\{\hat{V}_{j}\right\}$. These four modal parameters constitute the basis of the present shunt tuning procedure [23], which is verified in the following by experimental analysis.

The derivation of the tuning procedure applies a mixed modal projection of the $\mathrm{SC}$ and $\mathrm{OC}$ mode shapes, which expresses the effective EMCC

$$
\kappa_{j}^{2}=\frac{\hat{\omega}_{j}^{2}-\omega_{j}^{2}}{\omega_{j}^{2}}=\frac{\left\{Q_{j}\right\}^{t}\left\{\hat{V}_{j}\right\}}{\tilde{m}_{j} \omega_{r}^{2}}
$$

in terms of the modal charge, modal voltage and an intermediate modal mass $\tilde{m}_{j}=\left\{U_{j}\right\}^{t}[M]\left\{\hat{U}_{j}\right\}$, which is approximately equal to the SC modal mass $m_{j}=\left\{U_{j}\right\}^{t}[M]\left\{U_{j}\right\}$ for most practical cases with a limited change in the mode shape because of the inherent SC to OC stiffness increase. The modal equations follow from a decoupling of the general vibration problem (1) by the modal representation $\{U\}=[U]\{v\}$ with the SC mode shapes $\left\{U_{j}\right\}$ contained as columns in the modal matrix $[U]$ and the modal coordinates $v_{j}$ collected in the vector $\{v\}$. Hereby, the upper mechanical equation in (1) uncouples as

$$
\left(\omega_{j}^{2}-\omega^{2}\right) v_{j}-\frac{\left\{Q_{j}\right\}^{t}}{m_{j}}\{V\}=0
$$

for all vibration modes $j=1,2, \ldots, N$, while the bottom electric equation in (1),

$$
\sum_{j=1}^{N}\left\{Q_{j}\right\} v_{j}=\{Q\}-\left[C_{p}\right]\{V\}
$$

still depends on the full range of modal coordinates $v_{j}$.

The influence from the non-resonant vibration modes on the optimum shunt tuning arises from the linear combination of modal charges in (9), which is undesirable to fully evaluate. Since (8) states that $v_{j}$ is proportional to the voltage $\{V\}$, the influence from the non-resonant vibration modes around a target mode $j=r$ may be approximated by an additional term to the blocked piezoelectric capacitance as

$$
\begin{aligned}
\left\{Q_{r}\right\} v_{r} & =\{Q\}-\left(\left[C_{p}\right]+\sum_{j \neq r}^{N} \frac{\left\{Q_{j}\right\}\left\{Q_{j}\right\}^{t}}{m_{j} \omega_{j}^{2}} \frac{\omega_{j}^{2}}{\omega_{j}^{2}-\omega^{2}}\right)\{V\} \\
& \simeq\{Q\}-\left[C_{r}\right]\{V\}
\end{aligned}
$$


TABLE 1. Pure and modified single-mode calibration expressions for series shunt $i$ components and effective EMCCs.

\begin{tabular}{|c|c|c|c|}
\hline 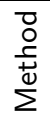 & $\begin{array}{l}\text { Inductance } \\
\qquad\left(L_{r}\right)_{i}\end{array}$ & $\begin{array}{l}\text { Resistance } \\
\qquad\left(R_{r}\right)_{i}\end{array}$ & $\begin{array}{l}\text { EMCC } \\
\kappa_{r}^{2}, \tilde{\kappa}_{r}^{2}\end{array}$ \\
\hline $\begin{array}{l}\frac{0}{60} \\
\stackrel{5}{5}\end{array}$ & $\frac{\left|\hat{V}_{r}\right|_{i}}{\left|Q_{r}\right|_{i} \hat{\omega}_{r}^{2}\left(1+\kappa_{r}^{2}\right)}$ & $\frac{\left|\hat{V}_{r}\right|_{i} \sqrt{2 \kappa_{r}^{2}}}{\left|Q_{r}\right|_{i} \hat{\omega}_{r}\left(1+\kappa_{r}^{2}\right)}$ & $\frac{\hat{\omega}_{r}^{2}-\omega_{r}^{2}}{\omega_{r}^{2}}$ \\
\hline 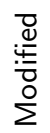 & $\frac{\left|\hat{V}_{r}\right|_{i}}{\left|Q_{r}\right|_{i} \hat{\omega}_{r}^{2}\left(1+\tilde{\kappa}_{r}^{2}\right)}$ & $\frac{\left|\hat{V}_{r}\right|_{i} \sqrt{2 \tilde{\kappa}_{r}^{2}}}{\left|Q_{r}\right|_{i} \hat{\omega}_{r}\left(1+\tilde{\kappa}_{r}^{2}\right)}$ & $\sum_{i=1}^{n_{p}} \frac{\left(Q_{r}\right)_{i}\left(\hat{V}_{r}\right)_{i}}{\left\{Q_{r}\right\}^{t}\left\{\hat{V}_{r}\right\}} \kappa_{r}^{2}$ \\
\hline
\end{tabular}

The introduced effective modal capacitance matrix $\left[C_{r}\right]$ is in the following assumed to be diagonal [23]. An effective modal capacitance is evaluated by considering the modal equations (8) and (10) in the OC limit and then introducing the alternative effective EMCC from (7). This results in the modal effective capacitance

$$
\left(C_{r}\right)_{i} \simeq-\frac{\left(Q_{r}\right)_{i}}{\left(\hat{V}_{r}\right)_{i}}
$$

for $i$ 'th piezoelectric absorber when $\tilde{m}_{j} \simeq m_{j}$ is assumed. Upon introduction of the impedance relation $\{V\}=-\mathrm{i} \omega[Z(\omega)]\{Q\}$ for the piezoelectric absorbers and the non-resonant modal approximation in (10), a governing characteristic equation for the target mode $r$,

$$
\begin{aligned}
&\left(\frac{\omega^{2}}{\omega_{r}^{2}}-\mathrm{i} \frac{\omega}{\omega_{r}} \sum_{i=1}^{N_{p}} \frac{\left(\hat{V}_{r}\right)_{i}\left(Q_{r}\right)_{i}}{\left\{\hat{V}_{r}\right\}^{t}\left\{Q_{r}\right\}} \frac{1}{Z_{i}(\omega)\left(C_{r}\right)_{i} \omega_{r}}\right) \\
& \times\left(1-\frac{\omega^{2}}{\omega_{r}^{2}}\right)+\kappa_{r}^{2} \frac{\omega^{2}}{\omega_{r}^{2}}=0
\end{aligned}
$$

is then obtained from (8). In the present analysis the series $L R$ shunt with $Z_{i}(\omega)=R_{i}+i \omega L_{i}$ is preferred because the intrinsic resistance of the passive inductors used in the experiments can then be directly accounted for by $R_{i}$ [14]. The modal characteristic equation is now obtained by substitution of $Z_{i}(\omega)=R_{i}+i \omega L_{i}$ into (12), from which the principle of equal modal damping [13] then determines the effective single-mode tuning expressions presented in Table 1 . The applied tuning procedure accounts for the influence from residual vibration modes directly by the modal voltage to charge ratio $\left|\hat{V}_{r}\right|_{i} /\left|Q_{r}\right|_{i}$ contained in the derived calibration expressions [23].

When a subset $n_{p} \leqslant N_{p}$ of all piezoelectric absorbers targets a specific vibration mode $j=r$, the presented single-mode calibration becomes non-optimal, as discussed in [23]. The limited authority of the reduced number of absorbers can however be represented by a modified effective EMCC

$$
\tilde{\kappa}_{r}^{2}=-\sum_{i=1}^{n_{p}} \frac{\left(Q_{r}\right)_{i}\left(\hat{V}_{r}\right)_{i}}{\tilde{m}_{r} \omega_{r}^{2}}=\sum_{i=1}^{n_{p}} \frac{\left(Q_{r}\right)_{i}\left(\hat{V}_{r}\right)_{i}}{\left\{Q_{r}\right\}^{t}\left\{\hat{V}_{r}\right\}} \kappa_{r}^{2}
$$

which mainly alters the optimum shunt resistance, as seen from the tuning formluas in the bottom row of Table 1.

2.1. Numerical results. The performance of the presented shunt tuning strategy is initially demonstrated numerically for the experimental setup with a free beam and five pairs of piezoceramic patches. The four piezoceramic patch pairs are shunted for vibration damping, while the fifth piezoceramic patch pair is used to induce structural vibrations. Dimensions and material properties of the beam and the piezoceramic patches are provided in the section Experiments. For the present numerical analysis the commercial FE-software ANSYS is used, see [22] for details on the implementation. The beam is discretized by $90 \times 6 \times 1$ 3D Solid186 elements, while each piezoceramic patch is modeled by $6 \times 6 \times 13 \mathrm{D}$ Solid226 coupled field elements. The natural frequencies 
TABLE 2. Free beam with four piezoceramic patch pairs: Numerical SC and OC frequencies, effective EMCC, modal capacitances and optimum shunt components.

\begin{tabular}{lccccc}
\hline Flex. mode & 1 & 2 & 3 & 4 & 5 \\
\hline$f[\mathrm{~Hz}]$ & 160.8 & 439.8 & 856.5 & 1406.3 & 2086.6 \\
$\hat{f}[\mathrm{~Hz}]$ & 162.7 & 444.5 & 864.3 & 1419.1 & 2121.5 \\
$\kappa_{r}^{2}[\%]$ & 2.42 & 2.16 & 1.81 & 1.83 & 3.38 \\
\hline$\left(C_{r}\right)_{1}[\mathrm{nF}]$ & 31.1 & 31.2 & 31.3 & 31.3 & 3.05 \\
$\left(C_{r}\right)_{2}[\mathrm{nF}]$ & 31.1 & 31.2 & 30.3 & 31.7 & 30.8 \\
$\left(C_{r}\right)_{3}[\mathrm{nF}]$ & 31.1 & - & 31.3 & - & 31.0 \\
$\left(C_{r}\right)_{4}[\mathrm{nF}]$ & 31.1 & 31.3 & 32.9 & 30.5 & 31.1 \\
\hline$\left(L_{r}\right)_{1}[\mathrm{H}]$ & 30.0 & 4.02 & 1.06 & 0.395 & 0.178 \\
$\left(L_{r}\right)_{2}[\mathrm{H}]$ & 30.0 & 4.02 & 1.09 & 0.389 & 0.177 \\
$\left(L_{r}\right)_{3}[\mathrm{H}]$ & 30.0 & - & 1.06 & - & 0.176 \\
$\left(L_{r}\right)_{4}[\mathrm{H}]$ & 30.0 & 4.01 & 1.01 & 0.405 & 0.175 \\
\hline$\left(R_{r}\right)_{1}[\mathrm{k} \Omega]$ & 6.74 & 2.33 & 1.10 & 0.674 & 0.617 \\
$\left(R_{r}\right)_{2}[\mathrm{k} \Omega]$ & 6.74 & 2.33 & 1.13 & 0.664 & 0.612 \\
$\left(R_{r}\right)_{3}[\mathrm{k} \Omega]$ & 6.74 & - & 1.10 & - & 0.608 \\
$\left(R_{r}\right)_{4}[\mathrm{k} \Omega]$ & 6.75 & 2.33 & 1.05 & 0.692 & 0.607 \\
\hline
\end{tabular}

and corresponding tuning parameters are for the lowest six modes determined by evaluation of the SC and OC eigenvalue problems in (3) and (5), respectively. The natural frequencies, effective modal capacitance (11) and series shunt components are summarized in Table 2. In the following section Experiments, the experimental shunt tuning results are compared by a percentage deviation to these numerical benchmark values. It should be noted that only the flexural vibration modes are considered in Table 2.

2.1.1. Modal load. For the present free beam example the four piezoelectric absorbers target the flexural vibration modes 2 to 5 , with the curves for the frequency response functions (FRFs) obtained by a full harmonic analysis of the discretized structure in ANSYS. Initially, the ideal modal load case $\left\{f_{r}\right\}=[M]\left\{U_{r}\right\}$ is considered to verify the equal modal damping principle used to derive the present shunt tuning method in Table 1. The FRF around the four target flexural vibration modes 2 to 5 can be seen in Figure 2(a,c,e,g) for the structure with the piezoelectric absorbers in OC condition (black dashed curves), with pure $L$-shunts (blue curves) and with optimum series $L R$-shunts (red curves). For each vibration mode $r=2$ to 5 in Figure 2, all four piezoelectric absorbers $i=1$ to 4 are calibrated specifically to that mode, as seen from the corresponding absorber voltage response in Figure 2(b,d,f,h).

Figure 2(a,c,e,g) show that the vibration reduction provided by the $L R$-shunted piezoelectric absorbers (red curves) agrees well with the dynamic amplification factor $1 /\left(2 \zeta_{r}\right)$, estimated by the modal damping ratio $\zeta_{r}=\sqrt{1 / 8 \kappa_{r}^{2}}$ from [23] with the effective EMCC $\kappa_{r}^{2}$ given in Table 1. Further remarkable is the intersections between the black, blue and red curves in Figure 2(a,c,e,g) which defines two damping independent frequencies, while the blue curve for the pure series $L$-shunt is seen to go to zero for $\omega=\omega_{r}\left(1+\kappa_{r}^{2}\right)$, which is the inverse point to the two corresponding split mode frequencies $\omega_{-}$and $\omega_{+}$in [22]. The response curves in Figure 2(a,c,e,g) are not completely flat because the equal modal damping calibration [13] implies a slight declination towards higher frequencies. However, the equal modal damping principle secures a fully flat plateau in absorber response amplitude with a unit magnitude when normalized appropriately by the applied modal load $\left\{f_{r}\right\}$. Figure $2(\mathrm{~b}, \mathrm{~d}, \mathrm{f}, \mathrm{h})$ verifies this condition, as the absorber voltage amplitudes at each resonance are exactly flat and add up to unity.

2.1.2. Electromechanical point load. In the experimental setups in the section Experiments a pair of piezoceramic patches is placed locally on the structure to excite structural vibrations. This load is modeled in the ANSYS model by applying an electric potential on the non-grounded interface 
(a)

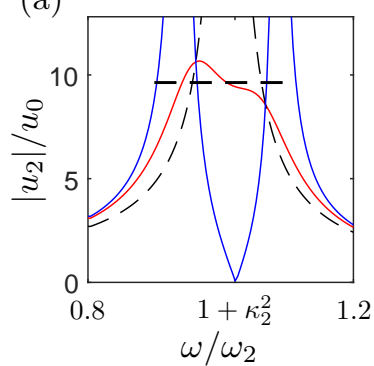

(c)

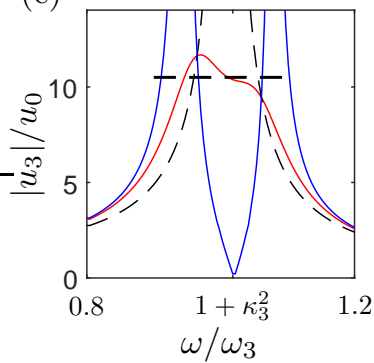

(e)
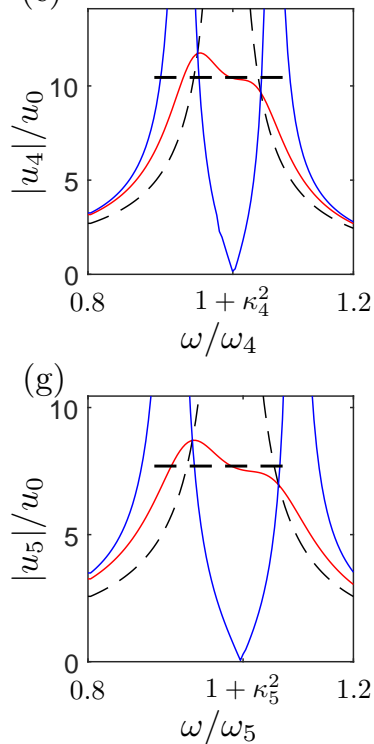

(b)

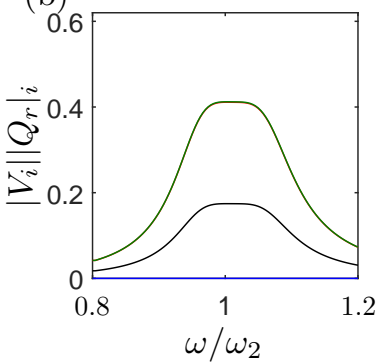

(d)

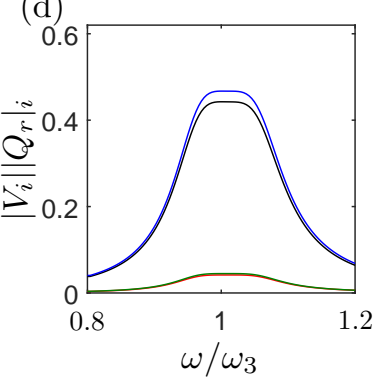

(f)
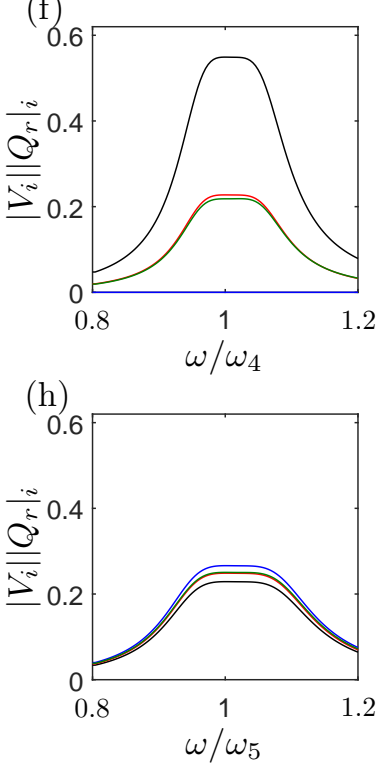

FiguRE 2. FRFs for free beam with 4 piezoelectric absorbers and modal load. Displacements at maximum modal deflection and voltages for damping of mode $r=2(\mathrm{a}, \mathrm{b}), 3(\mathrm{c}, \mathrm{d}), 4(\mathrm{e}, \mathrm{f})$ and $5(\mathrm{~g}, \mathrm{~h})$. (a,c,e,g): black, blue and red curves are FRFs for OC condition, $L$ - and $R L$-shunted absorbers, respectively, while the horizontal dashed lines represents $1 /\left(2 \zeta_{r}\right)$. (b,d,f,h): black, red, blue and green curves represent voltage amplitude curves absorbers 1 to 4 , respectively.

electrode of the fifth piezoceramic patch pair [22]. For this piezoelectric excitation, the response FRFs around the four target vibration modes 2 to 5 are shown in Figure 3(a,c,e,g) for the tip beam displacements with the piezoelectric absorbers in OC condition (black dashed curves), with pure $L$-shunts (blue curves) and with the optimum $L R$-shunts (red curves). The corresponding voltage response amplitude curves are shown in Figure 3(b,d,f,h) for the absorbers 1 to 4 .

For the electromechanical point load imposed by the fifth piezoelectric patch pair, the FRFs for the voltage response in Figure 3(b,d,f,h) are not entirely flat and thus non-optimal, while the tip displacement FRFs (red curves) in Figure 3(a,c,e,g) do not recover the precise inclination, 

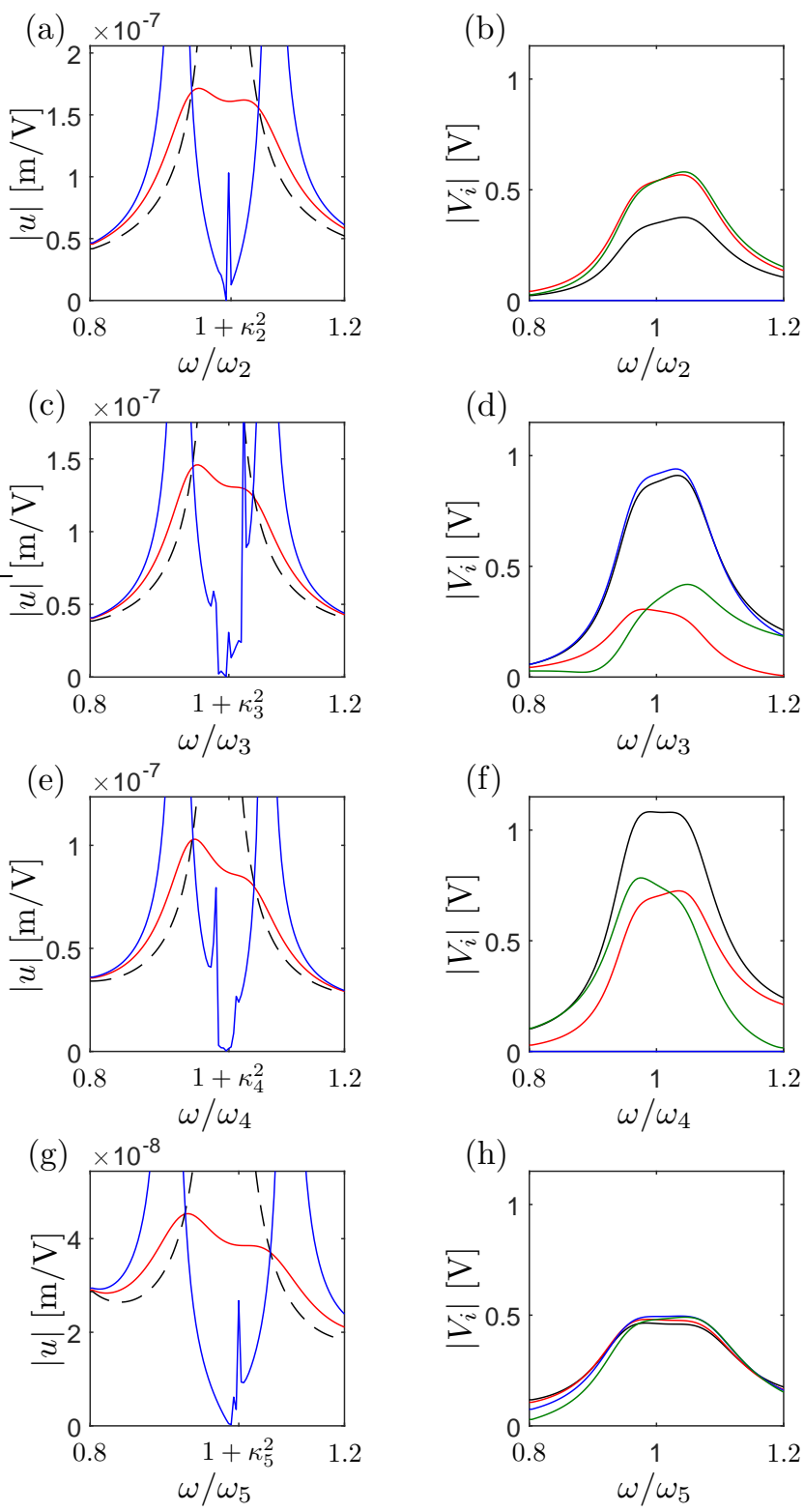

FiguRE 3. FRFs for free beam with 4 piezoelectric absorbers and electromechanical point load. Tip displacements and voltages for damping of mode $r=2(\mathrm{a}, \mathrm{b})$, $3(\mathrm{c}, \mathrm{d}), 4(\mathrm{e}, \mathrm{f})$ and $5(\mathrm{~g}, \mathrm{~h})$. (a,c,e,g): black, blue and red curves are FRFs for OC condition, $L$ - and $R L$-shunted absorbers, respectively, while the horizontal dashed lines represents $1 /\left(2 \zeta_{r}\right)$. (b,d,f,h): black, red, blue and green curves represent voltage amplitude curves absorbers 1 to 4 , respectively.

presented for the ideal modal load case in Figure 2(a,c,e,g). However, as the absorber voltage amplitudes (b,d,f,h) are almost flat and the displacement amplitudes (a,c,e,g) are effectively reduced, the performance is practically optimal, even for this specific load case that inherently activates other non-targeted vibration modes. The numerical results in Figure 3 constitute an important benchmark for the assessment of the actual experimental results presented in the section Experiments. 


\section{EXPERIMENTAL SHUNT TUNing}

This section describes the experimental implementation of the numerical shunt tuning methods from the previous section. The experimental method follows the approach of the numerical implementation, in which the modal parameters are evaluated in the two limiting situations associated with SC and OC piezoelectric electrodes. The structure is excited by the fifth piezoelectric patch pair with a pseudo-random vibration signal within a given frequency interval and a certain intensity. In terms of noise, it has been found that this type of signal appears superior to other random excitation methods. For each shunt the electric current $\mathrm{I}(t)$ and the voltage $\mathrm{V}(t)$ response signals are then measured for both SC and OC conditions. The measurements are acquired over a sufficiently long time-span and fast sampling rate to gather sufficiently detailed experimental FRFs by a spectrum analyzer to obtain the natural frequencies and electrical amplitudes to be used in the tuning procedure. The FRF for the electric charge is obtained from the measured FRF for the electric current by simply applying (2). Thus, the required modal shunt tuning parameters are directly obtained from the resonant peaks in the charge and voltage FRFs, which are available from the shunt terminals without any assessment of the structural response.

3.1. Influence of residual mode correction. The ratio of modal charge to voltage used (11) to estimate the effective modal capacitance may be determined experimentally by the ratio between the peaks in the charge and voltage FRFs. The effective capacitance is the main shunt tuning parameter and in particular a precise inductance tuning is crucial for the final damping performance of the piezoelectric absorber. For the series shunt inductance, the optimal tuning expression from Table 1 can be written in the traditional format

$$
\left(L_{r}\right)_{i}=\frac{1}{\left(C_{r}\right)_{i} \hat{\omega}_{r}^{2}\left(1+\kappa_{r}^{2}\right)}
$$

for which any tuning error is comprised by the modal capacitance $\left(C_{r}\right)_{i}$. For the traditional inductance tuning, based on a single-mode approximation without residual mode correction, the inductance is obtained by the similar expression

$$
\left(L_{r}^{S}\right)_{i}=\frac{1}{\left(\bar{C}_{p}^{S}\right)_{i} \hat{\omega}_{r}^{2}\left(1+\kappa_{r}^{2}\right)}
$$

where the effective modal capacitance is simply replaced by the resulting blocked capacitance $\left(\bar{C}_{p}^{S}\right)_{i}$, which is usually evaluated from the stress-free piezoelectric capacitance $C_{p}^{T}$ and a specific coupling coefficient $k$ provided by the manufacturer,

$$
C_{p}^{S}=C_{p}^{T}\left(1-k^{2}\right)
$$

This resulting blocked capacitance is the actual blocked capacitance for a single piezoelectric element, while it depends on the specific wiring between several piezoelectric patches connected to a single shunt.

As mentioned in the Introduction, the literature describes the difference between the blocked capacitance $C_{p}^{S}$ and a static capacitance $C_{p}^{0}$, measured at low frequencies when the piezoelectric element is bonded to the structure. The static capacitance is inherently larger than the blocked capacitance, yet smaller than the stress-free capacitance $C_{p}^{S}<C_{s t a t}<C_{p}^{T}$, as the boundaries of the piezoelectric element bonded to a flexible structure are only partially constrained in reality $[17,15]$. For the shunt tuning to lower resonance frequencies this static capacitance will therefore provide a more accurate inductance tuning by

$$
\left(L_{r}^{0}\right)_{i}=\frac{1}{\left(C_{p}^{0}\right)_{i} \hat{\omega}_{r}^{2}\left(1+\kappa_{r}^{2}\right)}
$$

while the use of the blocked capacitance may instead be more accurate for damping of very highfrequency modes, since $\left(C_{r}\right)_{i} \rightarrow\left(\bar{C}_{p}^{S}\right)_{i}$ for $\omega \rightarrow \infty[15]$.

A common practice in experimental piezoelectric shunt tuning has been to determine an optimum inductance tuning based on either the blocked or the static capacitance, while the final fine tuning has been performed by implementing the pure $L$-shunt and ensuring that the two split mode frequencies match the inverse frequency relation to the original target frequency [21]. This 
(a)

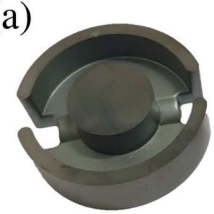

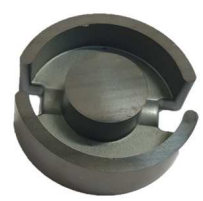

(b)

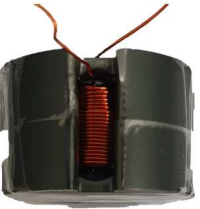

FiguRE 4. Passive inductor: (a) RM-core parts and (b) assembled inductor with $n$ wire turns around center coil.

has been a feasible method as the adjustable synthetic inductor [5] in general has been used to emulate the required shunt inductance. However, recently it has been demonstrated that the often required high inductance values may in fact be realized passively by winding a cobber wire around a magnetic core [14]. Whereas this provides a promising opportunity for the design of pure passive $L R$-shunts, it obviously eliminates the possibility to fine tune and therefore requires a very precise prior inductance tuning and thereby the number of wire turns in the passive inductor. Thus, it is the aim of this paper to demonstrate that the effective modal capacitance $\left(C_{r}\right)_{i}$ in (11) is secures an adequate and robust inductance tuning from (14) for the design of the passive inductor.

3.2. The passive inductor. This section summarizes the main properties of and practical experiences with the design of the passive inductors introduced in [14], to which the reader is referred for further details. In the present application a so-called RM-core is considered, see Figure 4. It consists of two parts Figure 4(a) that are assembled around a coil with $n$ wire turns to create the passive inductor shown in Figure 4(b). The passive inductor is designed to obtain the required inductance properties in an electric circuit, defined by the following ratio between the voltage and the rate of current,

$$
L=\frac{V}{\omega I}
$$

The voltage $V$ may also be described in the frequency domain by the total flux $\varphi$ through the $n$ turns of the coil $V=n \omega \varphi$. The magnetic flux will then follow from the current as $\varphi=n \mu\left(A_{e} / l_{e}\right) I$, where the effective permeability $\mu$, the magnetic cross section $A_{e}$ and the magnetic patch length $l_{e}$ are properties provided by the core manufacturer. By combining these expressions, the inductance of the wounded coil becomes proportional to the square of the $n$ turns,

$$
L=A_{L} n^{2} A \quad, \quad A_{L}=\frac{\mu A_{e}}{l_{e}}
$$

with $A_{L}$ being the permeance or constant inductance factor.

It is a challenge in the manufacturing of passive inductors to achieve a tight and proper winding arrangement. For this purpose a CNC Guitar Pickup Mini Coil Winder has been used, which permits a sufficient fine adjustment of coil and wire size and winds the exact number of desired turns. Once the desired coil wire arrangement is achieved, it is furthermore important to fully clamp the RM-core to the coil. Even small discrepancies in the clamping has been observed to significantly deteriorate the achieved inductance. It is strongly advised to the purchase RMcores with a center hole that enables the use of adjustment screws for the fine-tuning of the inductance. In the present experimental setup, the RM-cores in Figure 4(a) are without a center hole, whereby adequate fine-tuning has been performed by use of scotch and strips. Furthermore, an exact measurement of the realized inductance is important, as this may vary with frequency [14], temperature and magnitude of the current. In the present case, the inductance has been measured by considering the voltage-current relation for the electric circuit inductor (18). By applying a suitable alternating voltage between the poles of the inductor, while measuring the flow of current with a high-quality multimeter, the achieved inductance is determined precisely for the specific excitation frequency. Figure 5(a) shows a measured electric current to a specific voltage excitation for the RM-core T38 with material properties provided in Table 3 and $n=400$ wire turns. The theoretical inductance for these properties are $L=A_{L} n^{2}=4.48 \mathrm{H}$, while the actual inductance obtained by measurements with (18) is shown in Figure 5(b) as function of the 

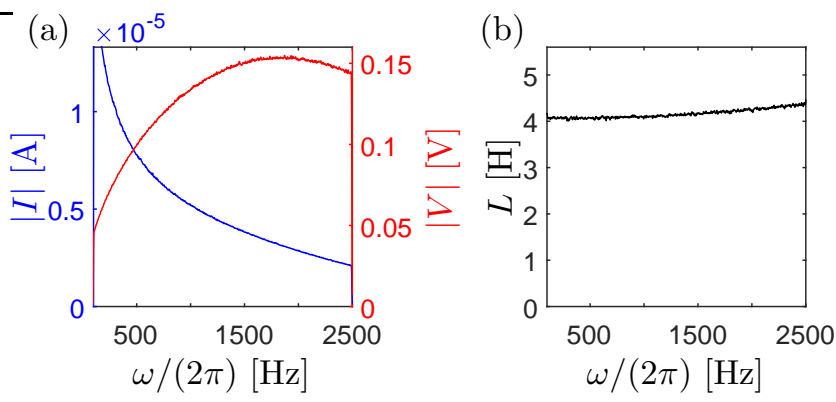

FIGURE 5. Effective inductance of passive inductor: (a) voltage excitation (red curve) and current response (blue curve) give effective inductance in (b).

TABLE 3. Geometry and properties of magnetic core.

\begin{tabular}{lccccc}
\hline RM-core & $A_{e}\left[\mathrm{~mm}^{2}\right]$ & $l_{e}[\mathrm{~mm}]$ & $B_{\text {sat }}[\mathrm{T}]$ & $\left.\mu_{e}[]^{-}\right]^{*}$ & $A_{L}[\mu \mathrm{H}]$ \\
\hline T38 & 145 & 46 & 0.43 & 7070 & 28 \\
\hline${ }^{*} \mu_{e}=\mu / \mu_{0}$ with vacuum permeability $\mu_{0}=0.4 \pi \mu \mathrm{H} / \mathrm{m}$
\end{tabular}

excitation frequency. It is found that the measured inductance is slightly lower than the theoretical estimate, corresponding to an inductance factor $A_{L} \simeq 25 \mu \mathrm{H}$ over the frequency interval of interest that is smaller than the theoretical value $(28 \mu \mathrm{H})$ in Table 3. Details on practical limitations of the passive inductors can be found in [14].

\section{EXPERIMENTS}

Experimental piezoelectric shunt damping of two plate-like structures is analyzed in the present section. The general experimental setup can be seen in Figure 6(a), while Figure 6(b,c) shows the free beam and the free plate structure, each with five pairs of piezoceramic patch pairs. In both experimental setups the fifth piezoceramic patch pair is connected to a power amplifier, which is controlled by the Laser Doppler Vibrometer acquisition software Polytec. The acquisition of the velocities measured by the Laser Doppler Vibrometer is also controlled by the Polytec acquisition sotfware, whereby FRFs and mode shapes of the tested structures are readily available. The shunt tuning of the piezoelectric absorbers is based on FRFs for current and voltage, as discussed in the section Experimental shunt tuning. These FRFs are obtained by a 34465A Keysight Digital Multimeter, see Figure 6(a), which supports high-resolution acquisition of the direct current and voltage time histories. The precise geometries and material properties for the free beam and plate structures are presented in the following subsections. The free beam is primarily used to validate the numerical results obtained with the ANSYS 3D FE-model presented in the section Numerical shunt tuning, while multi-modal damping of closely spaced vibration modes is addressed by the subsequent free plate experiment.

4.1. Free beam. The experimental results for the free beam with five pairs of piezoceramic patches in Figure 6(b) are presented and discussed in the present section. To realize free boundary conditions, the beam is hanging in two rubber bands (Figure 6(b)). The beam dimensions are $450 \times 30 \times 2 \mathrm{~mm}^{3}$ and the material is aluminum with a Young's modulus $E_{b}=71 \mathrm{GPa}$, Poison's raio $\nu_{b}=0.33$ and density $\rho_{b}=2710 \mathrm{~kg} / \mathrm{m}^{3}$. The five piezoceramic patches all have the same dimensions $30 \times 30 \times 6 \mathrm{~mm}^{3}$ and they are bonded to the beam with their center points placed in the one-sixth locations along the beam. The piezoceramic material is PZT 3265HD, purchased from [4], with full 3D material properties presented in Table 4. The free capacitance $C_{p}^{T}$ and in-plane coupling coefficient $k_{p}$ for each piezoceramic patch have furthermore been provided by the manufacturer, while the mean values used in the experimental analysis are given in Table 4. 
(a)

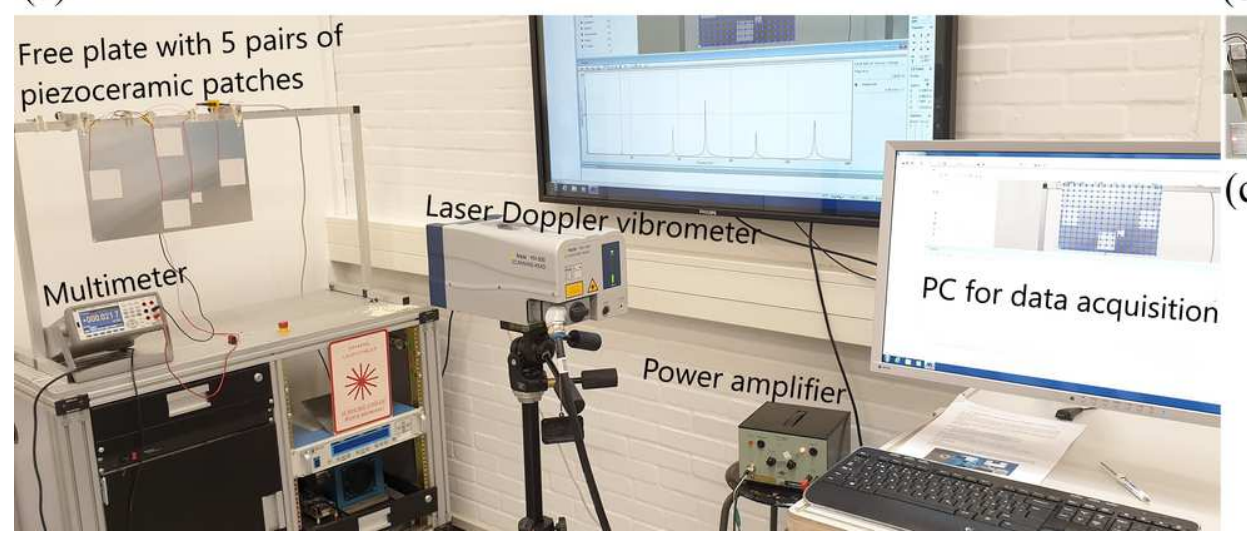

(b)

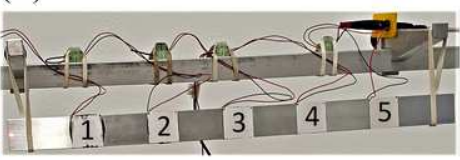

(c)

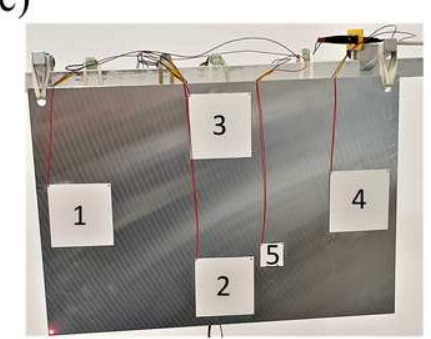

Figure 6. (a) Experimental setup and close-up of the tested free beam (b) and free plate (c), both with five pairs of piezoceramic patch pairs (1-5). Red points indicate location of laser deflection measurement at left beam tip in (b) and bottom left plate corner in (c).

For each pair the two piezoceramic patches are glued with non-conductive EPO-TEK 320 epoxy to both sides of the sanded aluminum beam, followed by a one hour curing at $130^{\circ} \mathrm{C}$. The nonconductive adhesive is used to avoid the risk of spill-over epoxy short-circuiting the electrodes of the thin piezoceramic patches. However, as each patch pair must be connected in series, the two inner electrodes must be electrically connected. This is ensured by applying a sufficient mechanical pressure during the curing to establish an electric connection between the inner electrodes and the aluminum beam, which then acts as the conductor between the two piezoceramic patches. Multi-filament cables are then soldered to the two outer piezoelectric electrodes, which can then be connected to the electric shunt. When bonded to the structure, the resulting static capacitance $\left(C_{p}^{0}\right)$ of each pair of piezoceramic patches may be determined by an LCR-meter. The static capacitance may also be evaluated numerically by applying a unit static voltage to each pair of piezoceramic patches in the FE code ANSYS, while the corresponding charge (capacitance per unit voltage) is obtain as the corresponding electric response. Both the experimental and numerical (ANSYS) static capacitances $C_{p}^{0}$ are provided in Table 4. As previously discussed in the section Experimental shunt tuning, the $C_{p}^{0}$ appears between the free $\left(\bar{C}_{p}^{T}\right)$ and the blocked $\left(\bar{C}_{p}^{S}\right)$ capacitances, with $(\overline{)})$ denoting the resulting capacitance from the series connection of the two patches in each piezoceramic pair.

4.1.1. Experimental shunt tuning. The experimental shunt tuning method presented in the section Experimental shunt tuning is now applied, by initially imposing a pseudo-random vibration excitation to the free beam, while the piezoceramic patch pairs 1 to 4 are in turn placed in SC and OC conditions. In the present case the considered frequency range of the pseudo-random excitation is $100-2500 \mathrm{~Hz}$, while the excitation intensity is set to $2 \mathrm{~V}$. By use of a multimeter, the corresponding SC current and OC voltage time histories are measured between the outer electrodes of the piezoceramic patch pairs 1 to 4 , with a sampling interval of $1 /(2 \times 2500 \mathrm{~Hz})=200 \mu$ s for a total of $2^{20}$ samples. The Matlab build-in spectrum generator pspectrum is then used on the electric current and voltage time series to obtain the corresponding FRFs. Figure 7(a-d) shows the FRFs for the OC voltage $V$ and SC charge $Q$ for the piezoelectric absorbers 1 to 4 . The charge FRF is obtained from the measured FRF of the electric current by the relation in (2).

The FRFs of the SC charge and OC voltage in Figure 7 for the piezoelectric absorbers 1 to 4 contain all the information needed for conducting the shunt tuning to any target vibration mode in the analyzed frequency spectrum. It can immediately be seen that five resonant vibration modes may be targeted in the analyzed frequency spectrum, for which the modal charge and voltage are determined as the peak resonance values indicated in Figure 7 by the vertical dashed blue and red 
TABLE 4. Stiffness components, stress piezoelectric coupling coefficients and blocked dielectric constants for PZT 3265HD [4].

\begin{tabular}{|c|c|c|c|}
\hline \multicolumn{2}{|c|}{ Stiffness components [GPa] } & \multicolumn{2}{|c|}{ Piezoelectric coupling $\left[\mathrm{C} / \mathrm{m}^{2}\right]$} \\
\hline$C_{11}^{E}, C_{22}^{E}$ & 151 & $e_{31}, e_{32}$ & -16.5 \\
\hline$C_{33}^{E}$ & 137 & $e_{33}$ & 30.3 \\
\hline$C_{12}^{E}$ & 99.0 & $e_{24}, e_{15}$ & 21.7 \\
\hline$C_{13}^{E}, C_{23}^{E}$ & 98.0 & \\
\hline$C_{44}^{E}, C_{55}^{E}$ & 24.2 & \multicolumn{2}{|c|}{$\begin{array}{l}\text { Blocked dielectric constants } \\
\varepsilon_{11}^{S}, \varepsilon_{0}^{S} \quad 2465 \varepsilon_{0}\end{array}$} \\
\hline$C_{66}^{E}$ & 26.0 & & $2100 \varepsilon_{0}$ \\
\hline \multicolumn{2}{|c|}{ Material density $\left[\mathrm{kg} / \mathrm{m}^{3}\right]$} & \multicolumn{2}{|c|}{ Plane coupling coefficient [-] } \\
\hline$\rho_{P Z T}$ & 8220 & $k_{p}$ & 0.66 \\
\hline \multicolumn{4}{|c|}{ Free, blocked and static capacitance (ANSYS) [nF] } \\
\hline $\bar{C}_{p}^{T}=C_{p}^{T} / 2$ & 46.7 & & \\
\hline $\bar{C}_{p}^{S}=\bar{C}_{p}^{T}\left(1-k_{p}^{2}\right)$ & 26.4 & & \\
\hline$C_{p}^{0}$ & 31.9 & & \\
\hline \multicolumn{4}{|c|}{ Free, blocked and static capacitance (Experiment) [nF] } \\
\hline $\bar{C}_{p}^{T}=C_{p}^{T} / 2$ & 47.2 & $k_{p}[-]$ & 0.54 \\
\hline $\bar{C}_{p}^{S}=\bar{C}_{p}^{T}\left(1-k_{p}^{2}\right)$ & 33.4 & & \\
\hline$\left(C_{p}^{0}\right)_{i}$ & 38.1 & 37.5 & 36.2 \\
\hline
\end{tabular}

(a)

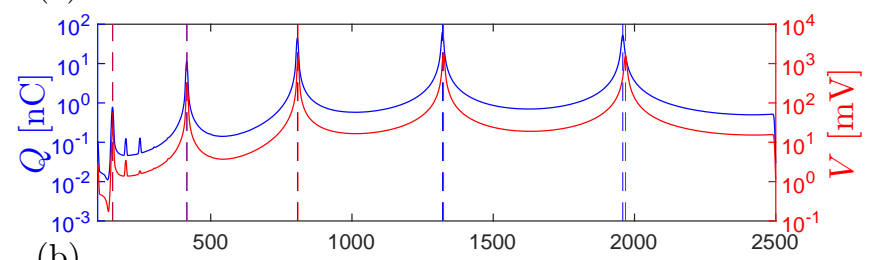

(b)

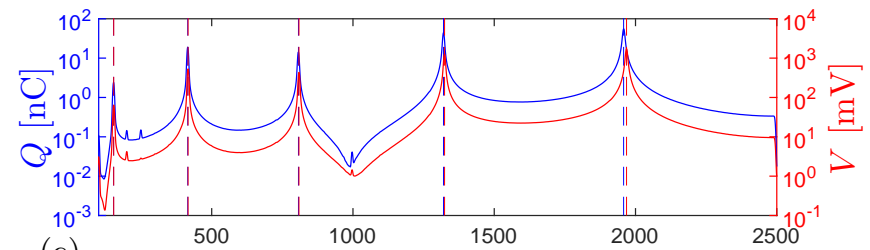

(c)

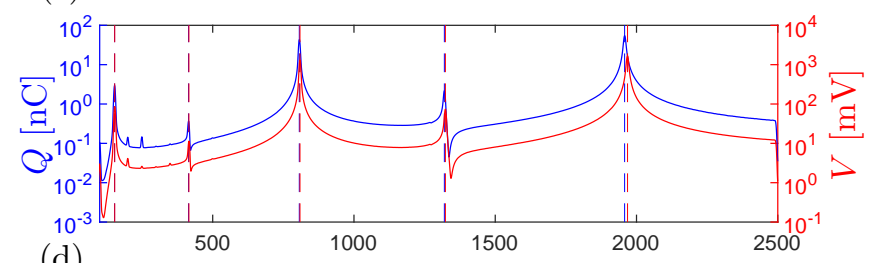

(d)

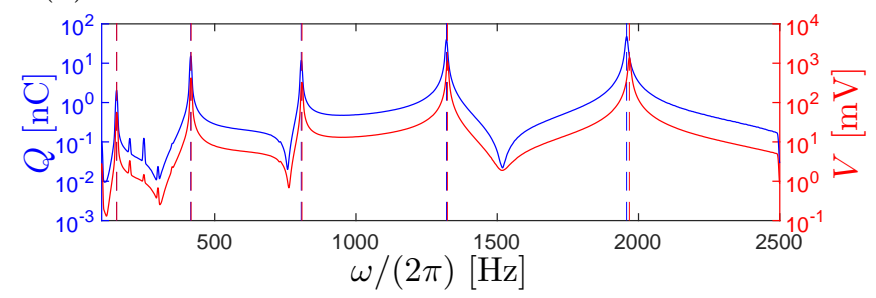

FiguRE 7. FRF for measured SC charge (blue curves) and OC voltage (red curves) for piezoelectric absorber 1-4(a-d). 
TABLE 5. Damping of free beam by four piezoceramic patch pairs: Experimental $\mathrm{SC}$ and $\mathrm{OC}$ frequencies, effective EMCC, modal capacitances and the optimum shunt tuning components based on charge and voltage FRFs in Figure 7.

\begin{tabular}{lccccc}
\hline Flex. mode & 1 & 2 & 3 & 4 & 5 \\
\hline$\omega /(2 \pi)[\mathrm{Hz}]$ & 152.6 & 415.1 & 806.8 & 1320.4 & 1958.0 \\
$\hat{\omega} /(2 \pi)[\mathrm{Hz}]$ & 153.2 & 416.4 & 808.9 & 1323.9 & 1968.1 \\
$\kappa_{r}^{2}[\%]$ & 0.80 & 0.59 & 0.53 & 0.56 & 1.03 \\
\hline$\left(C_{r}\right)_{1}[\mathrm{nF}]$ & 36.7 & 35.6 & 35.1 & 33.6 & 33.0 \\
$\left(C_{r}\right)_{2}[\mathrm{nF}]$ & 37.6 & 35.5 & 32.9 & 34.8 & 32.0 \\
$\left(C_{r}\right)_{3}[\mathrm{nF}]$ & 34.4 & 32.3 & 33.1 & 29.5 & 32.6 \\
$\left(C_{r}\right)_{4}[\mathrm{nF}]$ & 36.9 & 36.1 & 36.1 & 35.8 & 34.4 \\
\hline$\left(L_{r}\right)_{1}[\mathrm{H}]$ & 29.2 & 4.08 & 1.10 & 0.427 & 0.196 \\
$\left(L_{r}\right)_{2}[\mathrm{H}]$ & 28.5 & 4.09 & 1.17 & 0.413 & 0.202 \\
$\left(L_{r}\right)_{3}[\mathrm{H}]$ & 31.1 & 4.52 & 1.16 & 0.487 & 0.198 \\
$\left(L_{r}\right)_{4}[\mathrm{H}]$ & 29.0 & 4.02 & 1.05 & 0.402 & 0.185 \\
\hline$\left(R_{r}\right)_{1}[\mathrm{k} \Omega]$ & 3.55 & 1.16 & 0.532 & 0.375 & 0.344 \\
$\left(R_{r}\right)_{2}[\mathrm{k} \Omega]$ & 3.47 & 1.16 & 0.568 & 0.362 & 0.364 \\
$\left(R_{r}\right)_{3}[\mathrm{k} \Omega]$ & 3.80 & 1.28 & 0.651 & 0.427 & 0.358 \\
$\left(R_{r}\right)_{4}[\mathrm{k} \Omega]$ & 3.54 & 1.14 & 0.588 & 0.322 & 0.330 \\
\hline
\end{tabular}

lines, while the SC and OC frequencies are identified by the dashed lines intersections with the frequency axis. From the modal charges and voltage the corresponding effective capacitances are determined by (11) and provided in Table 5 . The magnitude of the individual modal charge and voltage furthermore reveal which piezoelectric absorbers are suitably targeting which vibration mode. It is seen that the third piezoelectric absorber in Figure 7(c) may not be used to damp modes 2 and 3, while a closer inspection of the resonance peak values in Figure 7(b,c) indicate that the piezoelectric absorbers 2 and 4 are a inadequate for damping of mode 3 . An overview of all tuning values for all targeting modes is provided in Table 5, with those in red constituting the optimal configuration.

As a supplement to the electric FRF, the physical response of the free beam has been measured using the Laser Doppler Vibrometer in Figure 6, which produces the experimental mode shapes in Figure 8. Fomr these mode shapes and the position of the piezoelectric absorbers 1-4 it is seen that the (optimal) red entries in Table 5 correspond to the cases, in which the specific patch pair is located at a nodal point of the mode shape associated with maximum curvature. Furthermore, it is seen that the homogeneous distribution of the piezoelectric absorbers along the beam is particularly optimal for the electromechanical coupling of mode 5 in Figure 8(e), for which all absorbers experience maximum curvature.

When comparing the numerical results in Table 2 to the experimental results in Table 5 , it is seen that there is a noticeable deviation on the effective EMCC $\kappa_{r}^{2}$. This may be particularly due to the quality of the gluing of the piezoelectric patches to the aluminum beam, which is considered ideal in the numerical ANSYS model. The quality of the gluing is highly sensitive to small differences in the amount of epoxy and also to the pressure applied during the curing process. In the present case generally too much epoxy have been applied between patches and beam, whereby a considerable amount of strain energy may be stored in the epoxy layer. However, besides the deviation in effective EMCC, a good agreement is observed for the resonance frequencies, the effective modal capacitances and the shunt tuning components. For the higher vibration modes the deviation between the numerical model and the experimental results increases slightly, which might occur because more $\mathrm{FE}$ elements are required in the numerical model to obtain the same accuracy on the higher vibration modes. However, an advantage of the present experimental shunt tuning method is that the tuning is based entirely on the piezoelectric absorber response, whereby the bonding quality and eventual material imperfections are inherently included in the procedure. 
(a)

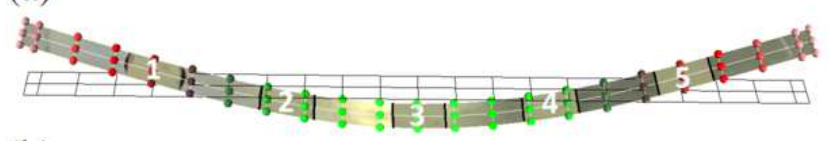

(b)

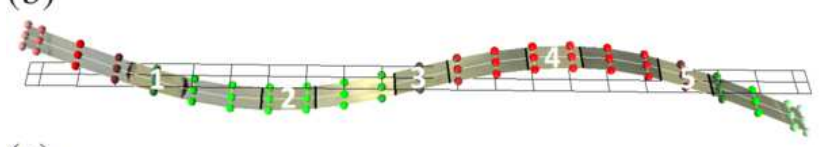

(c)

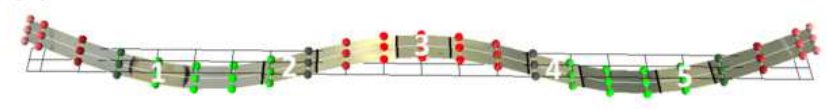

(d)

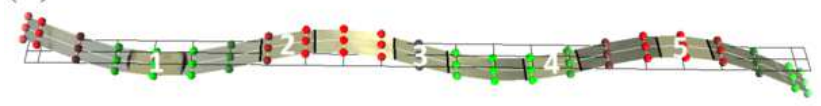

(e)

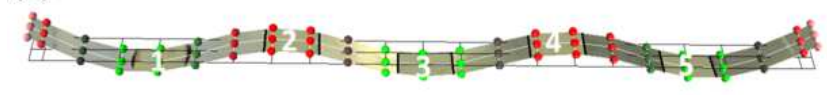

Figure 8. Experimental flexural mode shapes 1-5(a-e) of the free beam with five pairs of SC piezoceramic patches.

4.1.2. FRF analysis. The performance of the optimum shunt tuning is now analyzed by studying the FRFs based on the time response of the tip displacements, measured when the structure is excited with pseudo-random vibrations by the fifth piezoelectric patch pair. Four different damping cases are considered, in which all piezoelectric absorbers 1-4 are collectively shunted to one of the target modes 2, 3, 4 and 5, respectively. Each resonant shunt is designed by the passive inductor presented in the section The passive inductor, connected in series with a potentiometer permitting an easy adjustment of the shunt resistance. The passive inductors are designed to achieve inductance values as close to the predetermined optimum tuning as possible. The required number of wire turns $n$ are calculated by (19) with the constant attainable inductance factor $A_{L} \simeq$ $25 \mu \mathrm{H}$ measured for the RM-core T38. The inductors to the target vibration modes 2, 3, 4 and 5 are designed with $n=400, n=200, n=130$ and $n=90$ wire turns, respectively. The fine-adjustment for each piezoelectric absorber tuning is subsequently done by simply changing the tightness of the RM-core clamping, as discussed in the section The passive inductor. Finally, the obtained inductance values are measured using the method presented in the section The passive inductor, from which the mean value and relative standard deviation (Std) in a frequency interval (0.8 to 1.2) $\times \omega_{r}$ are determined and provided in Table 6. The deviation (Dev) between the exact tuning in Table 5 and the passively obtained inductance around the target modes is also provided in Table 6 , indicating a relatively good accuracy of the produced passive inductors.

The FRFs for the tip displacements around the target modes 2, 3, 4 and 5 with the piezoelectric absorber 1 to 4 shunted only by the passive inductors can be seen in Figure 9(a,c,e,g) by the blue solid curves, while the blacked dashed curves indicate the original OC response. Subsequently, the potentiometers are connected in series with the passive inductors and adjusted to the resistance values provided in Table 6 . It is seen from the relative deviation to the optimum resistance tuning in Table 5 that the implemented resistances are considerably lower than the optimum tuning. This is due to the inherent resistance of the passive inductor, which will not be analyzed in further detail. The FRFs for the tip displacements with the optimum $L R$-shunted absorbers can be seen in Figure $9(\mathrm{a}, \mathrm{c}, \mathrm{e}, \mathrm{g})$ by the red solid curves, while the corresponding absorber voltage responses for the piezoelectric absorbers 1 to 4 are provided in Figure 9(b,d,f,h).

As discussed in the section Numerical shunt tuning, the present shunt tuning method is based on the principle of equal modal damping, which results in slightly inclined plateaus in the FRF for the 
TABle 6. (Top) Passive inductances with relative standard deviations (Std) and (bottom) shunt resistance. Relative deviation (Dev) to desired optimum shunt tuning in Table 5 is provided.

\begin{tabular}{lcccc}
\hline Flex. mode & 2 & 3 & 4 & 5 \\
\hline$\left(L_{r}\right)_{1}[\mathrm{H}]$ & 4.09 & 1.07 & 0.428 & 0.193 \\
Std [\%] & $(0.43)$ & $(0.30)$ & $(0.39)$ & $(0.48)$ \\
Dev. & $0.31 \%$ & $-2.54 \%$ & $0.07 \%$ & $-1.96 \%$ \\
$\left(L_{r}\right)_{2}[\mathrm{H}]$ & 4.07 & - & 0.411 & 0.203 \\
Std [\%] & $(0.38)$ & - & $(0.42)$ & $(0.44)$ \\
Dev. & $-0.61 \%$ & - & $-0.56 \%$ & $0.39 \%$ \\
$\left(L_{r}\right)_{3}[\mathrm{H}]$ & - & 1.17 & - & 0.201 \\
Std [\%] & - & $(0.53)$ & - & $(0.32)$ \\
Deviation & - & $0.43 \%$ & - & $1.15 \%$ \\
$\left(L_{r}\right)_{4}[\mathrm{H}]$ & 4.02 & - & 0.400 & 0.186 \\
Std [\%] & $(0.32)$ & - & $(0.45)$ & $(0.46)$ \\
Dev. & $-0.06 \%$ & - & $-0.63 \%$ & $-1.56 \%$ \\
\hline$\left(R_{r}\right)_{1}[\Omega]$ & 750 & 250 & 250 & 250 \\
Dev. & $-35 \%$ & $-53 \%$ & $-33 \%$ & $-27 \%$ \\
$\left(R_{r}\right)_{2}[\Omega]$ & 750 & - & 250 & 250 \\
Dev. & $-35 \%$ & - & $-31 \%$ & $-31 \%$ \\
$\left(R_{r}\right)_{3}[\Omega]$ & - & 250 & - & 250 \\
Dev. & - & $-62 \%$ & - & $-3 \%$ \\
$\left(R_{r}\right)_{4}[\Omega]$ & 750 & - & 250 & 250 \\
Dev. & $-34 \%$ & - & $-22 \%$ & $-24 \%$ \\
\hline & & & &
\end{tabular}

displacements around the target resonant vibration modes, see numerical results in Figure 3(a,c,e,g). However, the method implies completely flat plateaus for the absorber voltage response in the case of and ideal modal load (Figure 2(b,d,f,h)) and almost flat plateau for an electromechanical point load case (Figure 3(b,d,e,f)). Therefore the validation of the experimental shunt tuning method is best performed by inspecting the absorber voltage response in Figure 9(b,d,e,f), from which it can be seen that the FRF for the 4 absorbers around the four target modes are relatively flat, with the exception of the absorber response around target mode 3 in Figure 9(d), in which the inductances for absorber 1 and 3 seem, respectively, too low and too high. However, this tendency is seen to agree with the deviation for the implemented passive inductors in the third column of Table 6. Small deviations from the optimum flat plateau may also be encountered for the other three target modes, but in all cases the tendency seem to follow the deviation between the optimum inductances in Table 5 and the implemented inductances in Table 6. By looking at the FRFs for the tip displacements with the $L R$-shunts implemented (red curves) in Figure 9(a,c,e,g) it is seen that these have the same form as those obtained in the numerical example in Figure 3. For target modes 2,3 and 4 in Figure 9(a,c,e) the plateau inclination is too large, while the opposite inclination for target mode 5 in Figure $9(\mathrm{~g})$ occurs because of a too large inductance. The present FRFs for the free beam experiment show that the proposed shunt tuning method, based on experimental FRFs for the charge and voltage signals from the four piezoelectric absorbers, appears robust as it approximately recovers the desired frequency response characteristics across the full frequency range, with deviations likely associated with inaccuracies in the produced passive inductances.

4.1.3. Inductance from blocked and static capacitance. As discussed in the section Experimental shunt tuning it is particular important to obtain a precise inductance tuning, as even small deviations may substantially detune the shunt absorbers and because the use of passive inductors will not allow any straightforward fine tuning. The inductance tuning in (17) and in (15) is based on the static capacitance $\left(C_{p}^{0}\right)_{i}$ and the blocked capacitance $\bar{C}_{p}^{S}$, respectively. These inductances in Table 7 are 

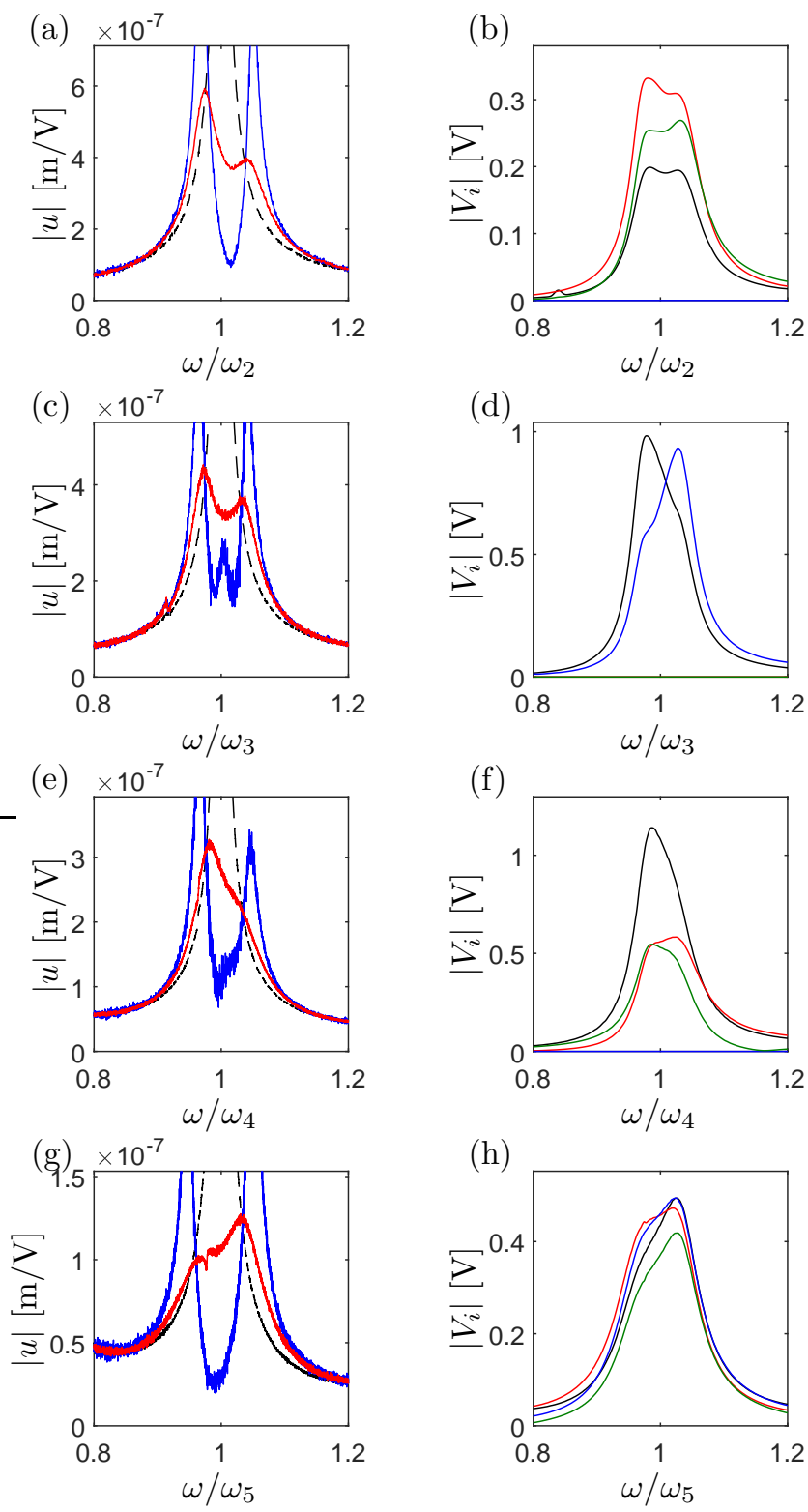

FiguRE 9. Experimental FRF for free beam with 4 piezoelectric absorbers and electromechanical point load. Tip displacements and voltages for damping of mode $r=2(\mathrm{a}, \mathrm{b}), 3(\mathrm{c}, \mathrm{d}), 4(\mathrm{e}, \mathrm{f})$ and $5(\mathrm{~g}, \mathrm{~h})$. (a,c,e,g): black, blue and red curves are FRFs for OC, $L$ - and $L R$-shunted absorbers, respectively. (b,d,f,h): black, red, blue and green curves represent voltage amplitude curves absorbers 1 to 4 , respectively.

determined based on the measured capacitances in Table 4 and used to illustrate the potential detuning associated with the exclusion of the residual mode contribution contained in the effective modal capacitance $\left(C_{r}\right)_{i}$ in (11). It can be seen from Table 7 that the inductance tuning based on the static capacitance $C_{p}^{0}$ is relatively accurate for the lower-frequency modes, while the blocked capacitance $C_{p}^{S}$ becomes better for target modes with higher frequencies. This agrees with the tendency of the dynamic capacitance, which approaches the blocked capacitance for increasing frequency. However, the use of either the static or the blocked capacitance for the inductance tuning may therefore cause errors in the high- or low-frequency limits, respectively. 
TABLE 7. Shunt inductance tuning based on the experimental static $\left(C_{p}^{0}\right)_{i}$ and blocked $\bar{C}_{p}^{S}$ capacitances (Table 4). Deviation (Dev) relative to the tuning in Table 5 based on effective modal capacitance $\left(C_{r}\right)_{i}$.

\begin{tabular}{lccccc}
\hline Flex. mode & 1 & 2 & 3 & 4 & 5 \\
\hline$\left(L_{r}^{0}\right)_{1}[\mathrm{H}]$ & 28.1 & 3.82 & 1.01 & 0.378 & 0.170 \\
$\left(L_{r}^{0}\right)_{2}[\mathrm{H}]$ & 28.5 & 3.87 & 1.03 & 0.383 & 0.172 \\
$\left(L_{r}^{0}\right)_{3}[\mathrm{H}]$ & 30.0 & 4.07 & 1.08 & 0.400 & 0.181 \\
$\left(L_{r}^{0}\right)_{4}[\mathrm{H}]$ & 29.0 & 4.02 & 1.06 & 0.397 & 0.179 \\
Mean Dev. [\%] & -1.3 & -5.4 & -6.6 & -9.3 & -10 \\
\hline$\left(L_{r}^{S}\right)_{i}[\mathrm{H}]$ & 32.0 & 4.35 & 1.15 & 0.430 & 0.194 \\
Mean Dev. [\%] & 8.7 & 4.1 & 2.7 & -0.6 & -1.4 \\
\hline
\end{tabular}
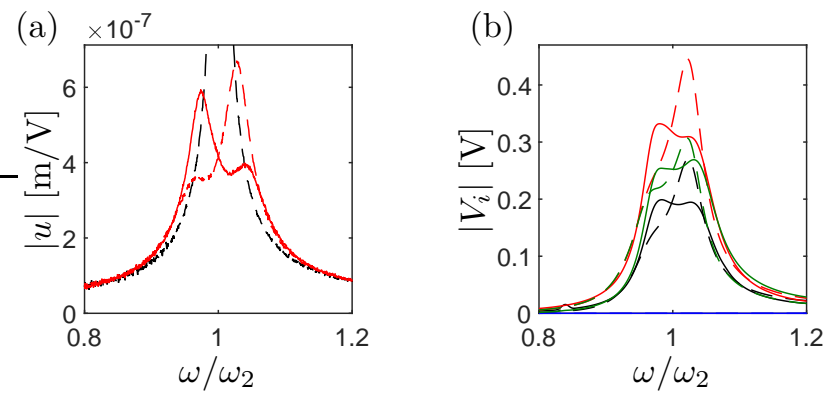

FiguRE 10. Experimental FRF for the free beam with 4 piezoelectric absorbers tuned with effective modal capacitance $\left(C_{r}\right)_{i}$ (red solid curves) and blocked capacitance $C_{p}^{S}$ (red dashed curves). Tip displacements (a) and the absorber voltage (b).

Figure 10 shows the FRF for the tip beam deflection (a) and shunt voltage (b) for target mode $r=2$. The red solid curve represents the present tuning based on the effective modal capacitance $\left(C_{r}\right)_{i}$, while the red dashed curve is for the associated blocked capacitance $C_{p}^{S}$. The passive inductors used in the experiment for the tuning based on the blocked capacitance are designed with $n=415$ wire turns, whereby the inductance values are $\left(L_{r}\right)_{1}=4.33 \mathrm{H},\left(L_{r}\right)_{2}=4.36 \mathrm{H}$ and $\left(L_{r}\right)_{4}=4.32 \mathrm{H}$, with a relative standard deviation to the numerical values of $0.46 \%, 0.43 \%$ and $0.51 \%$, respectively. The present tuning based on $\left(C_{r}\right)_{i}$ results in an increased inclination of the plateau in the response FRF in Figure 10(a), whereas the inductance tuning based on the blocked capacitance $C_{p}^{S}$ has an opposite inclination, indicating substantially detuning. For the voltage FRF in Figure 10(b) the present tuning method implies almost flat plateaus, while for the blocked capacitance tuning the dashed curves have substantial peaks.

4.2. Free plate. The second experiment concerns the free plate with five pairs of piezoceramic patches in Figure 6(c). The plate dimensions are $450 \times 300 \times 6 \mathrm{~mm}^{3}$ and it is made of aluminum with a Young's modulus $E_{b}=71 \mathrm{GPa}$, Poison's raio $\nu_{b}=0.33$ and material density $\rho_{b}=2710 \mathrm{~kg} / \mathrm{m}^{3}$. The piezoceramic material is the same as in the previous experiment with full $3 \mathrm{D}$ material properties provided in Table 4 . In the present setup the fifth pair of piezoceramic patches with dimensions $30 \times 30 \times 0.5 \mathrm{~mm}^{3}$ is used to excite structural vibrations, while the remaining pairs 1 to 4 with the dimensions $75 \times 75 \times 0.5 \mathrm{~mm}^{3}$ are shunted for vibration mitigation. The piezoelectric patch pairs 1 and 4 are centered along the vertical y-axis (short plate side), while their center is located respectively $50 \mathrm{~mm}$ from the left and right plate edges. Similarly, the piezoelectric patch pair 2 and 3 are centered along the horizontal $\mathrm{x}$-axis (long plate side), with the patch center located $50 \mathrm{~mm}$ from the bottom and top plate edges, respectively. Finally, the fifth piezoceramic patch pair is placed with its center at respectively $287.5 \mathrm{~mm}$ and $87.5 \mathrm{~mm}$ along the $\mathrm{x}$ and $\mathrm{y}$ directions from the 
TABLE 8. Free, blocked and static capacitance of the plate bonded piezoceramic patch pairs 1-4 Figure 6(c).

\begin{tabular}{|c|c|c|c|c|}
\hline \multicolumn{5}{|c|}{ Free, blocked and static capacitance (Experiment) [nF] } \\
\hline $\bar{C}_{p}^{T}=C_{p}^{T} / 2$ & 298 & $k_{p}[-]$ & & 0.62 \\
\hline $\bar{C}_{p}^{S}=\bar{C}_{p}^{T}\left(1-k_{p}^{2}\right)$ & 185 & & & \\
\hline$\left(C_{p}^{0}\right)_{i}$ & 254 & 245 & 233 & 252 \\
\hline
\end{tabular}

bottom left plate corner. All the piezoceramic patches are glued to the aluminum plate using the approach described for the free beam experiment in the previous subsection.

The free capacitance and in-plane piezoelectric coupling coefficient of the piezoceramic patches (pair 1 to 4) have been provided by [4], from which the resulting free $\bar{C}_{p}^{T}$ and blocked $\bar{C}_{p}^{S}$ capacitances for the series connected patch pairs are determined and summarized in Table 8. Furthermore, the static capacitances $\left(C_{p}^{0}\right)_{i}$ of the piezoceramic patch pairs 1-4 have been measured and provided in Table 8.

It can be seen that the four static capacitances $\left(C_{p}^{0}\right)_{i}$ in Table 8 appear as expected between the blocked $\bar{C}_{p}^{S}$ and free $\bar{C}_{p}^{T}$ capacitances. However, in the present case the static capacitances are in general closer to the free capacitance, which indicates a poorer bonding between the patches and the plate, whereby the patches become less constrained. The reason for the poor bonding is mainly due to insufficient pressure applied on to the fairly large piezoelectric patches during the curing process.

4.2.1. Experimental shunt tuning. The shunt tuning procedure in the section Experimental shunt tuning is now applied by initially exciting plate vibrations with a pseudo-random vibration signal in the frequency interval from $100-800 \mathrm{~Hz}$. The SC current and $\mathrm{OC}$ voltage response signals are then measured between the interface electrodes of the piezoelectric absorbers 1-4. The electric absorber response is measured with a sampling interval of $1 /(2 \times 800 \mathrm{~Hz})=625 \mu$ s for a total of $2^{19}$ samples. Subsequently, the build-in Matlab spectrum generator pspectrum is used on the absorber current and voltage time series to obtain the corresponding FRFs shown in Figure 11. The FRF for the charge is obtained by (2) from the FRF of the measured electric current.

It can be seen from the FRFs of the SC charge and OC voltage in Figure 11 that five resonant vibration modes are excited in the analyzed frequency interval. The vibration response of the plate is measured by the Laser Doppler Vibrometer and the first six modes are shown in Figure 12. It is found that the first free plate mode in Figure $12(\mathrm{a})$ at $f_{1}=149.5 \mathrm{~Hz}$ is not apparent in the FRFs for the measured current and voltage in Figure 11, since the four piezoelectric absorbers are either exposed to in-plane shear or located in a nodal point of the vibration mode in Figure 12(a).

The shunt tuning parameters for the vibration modes 2 to 6 are now determined directly from the FRFs for charge and voltage by their respective peak values, indicated by the vertical dashed lines in Figure 11, which also determine the corresponding SC and OC frequencies. The effective modal capacitances $\left(C_{r}\right)_{i}$ are then evaluated from the peak modal charge and voltage values according to (11). They are given in Table 9 together with the optimum series shunt tuning parameters. It is seen from the peak charge and voltage values that the absorber 1 and 4 in Figure 11(a,d) are not applicable for damping of modes 2 and 3, while the absorbers 2 and 3 in Figure 11(b,c) have very limited authority of modes 5 and 6 . An overview of all tuning values for all targeting modes is provided in Table 5 , in which those values in red constitute the chosen shunt configuration.

The passive inductors are designed according to the procedure in the section The passive inductor with the aim of achieving the optimum inductance tuning provided in Table 9 for the targeted modes 2, 3, 4 and 6 . These four modes are targeted because they all possess maximum modal displacements in the plate corners, whereby the effect of the damping can be analyzed from FRFs for the plate corner displacements, as indicated by the red laser point in Figure 6(c). For each of the four targeted modes the inductors emphasized in red in Table 9 have been wound (XXX winded?XX) with $n=420,450$ turns for mode $2, n=198,200$ turns for mode 3 , four times $n=180$ turns for mode 4 and $n=125,127$ turns for mode 6 . Table 10 provides the corresponding mean 
(a)
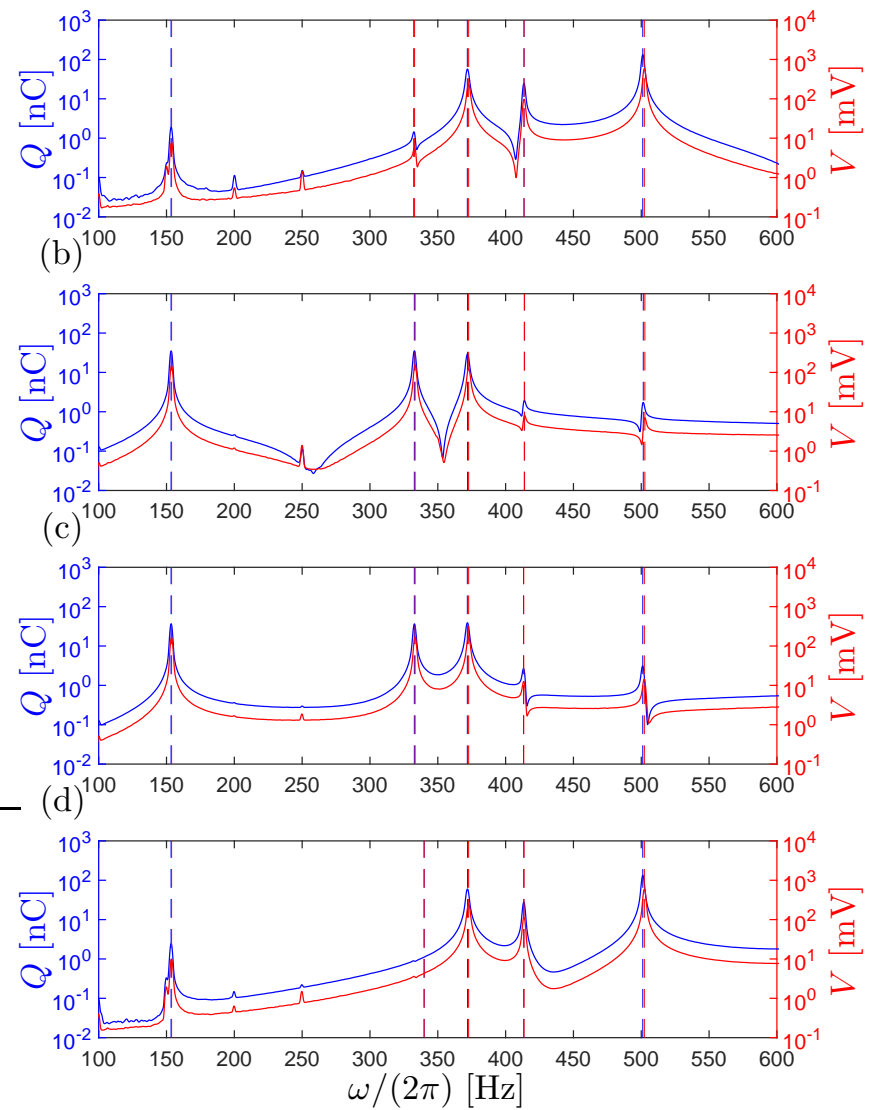

FiguRE 11. FRF for measured SC charge (blue curves) and OC voltage (red curves) for piezoelectric absorber 1-4(a-d).

inductance values around the target frequencies $\left(0.8 \omega_{r}-1.2 \omega_{r}\right)$, the relative standard deviation and the deviation relative to the optimum inductance tuning in Table 9.

4.2.2. FRF analysis. The deviations in Table 10 indicate that the produced passive inductors are in good agreement with the desired optimum shunt tuning in Table 9. Figure 13 shows the FRFs for the measured corner displacement of the free plate structure in (a,c,e,g) and the corresponding voltage across the patch electrodes in (b,d,f,h). The blue solid curves in Figure 13(a,c,e,g) represent the pure $L$-shunts for the absorbers 1 to 4 , which appear to have two well-separated peaks with a minimum close to $\omega / \omega_{j}=1$. When introducing the shunt resistance from Table 10 in in series with the passive inductors, the red curves in Figure 13(a,c,e,g) become almost flat around resonance. Furthermore, the absorber voltage response in Figure 13(b,d,f,h) is practically flat, which indicates a proper shunt tuning.

A pronounced resonance peak in the FRF for the plate corner displacement can be seen around the target mode 2 in Figure 13(a), which occurs because of the closely spaced resonant mode 1 in Figure 12(a), not damped by the piezoelectric absorbers. To avoid the influence of mode 1, the response for target mode 2 may instead be analyzed by considering the FRF for the displacements of the center point on the left plate edge, where mode 1 in Figure 12(a) has a nodal point. Figure 14(a) shows the FRF for this new measuring point, which exhibits a flat plateau around target mode 2 .

4.2.3. Inductance based on blocked and static capacitance. The influence of neglecting the residual mode contributions in the shunt inductance tuning is analyzed by tuning the inductances based on the static $\left(C_{p}^{0}\right)_{i}$ and blocked $C_{p}^{S}$ capacitances in Table 8. These inductance values are provided 
(a)

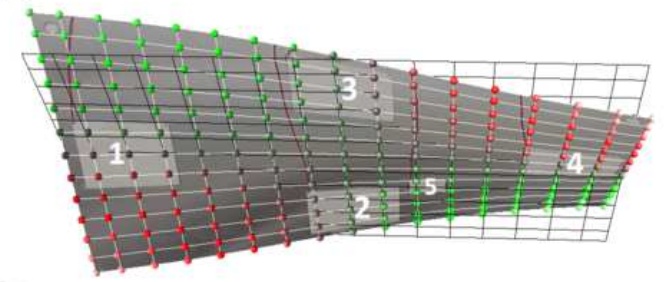

(b)

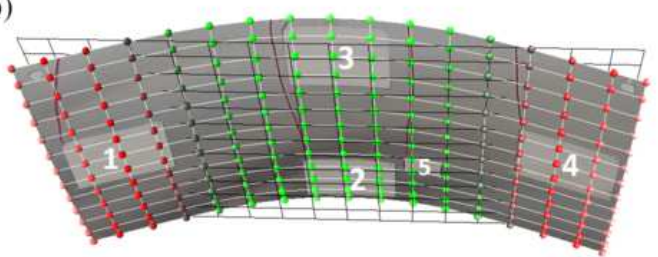

(c)

(d)
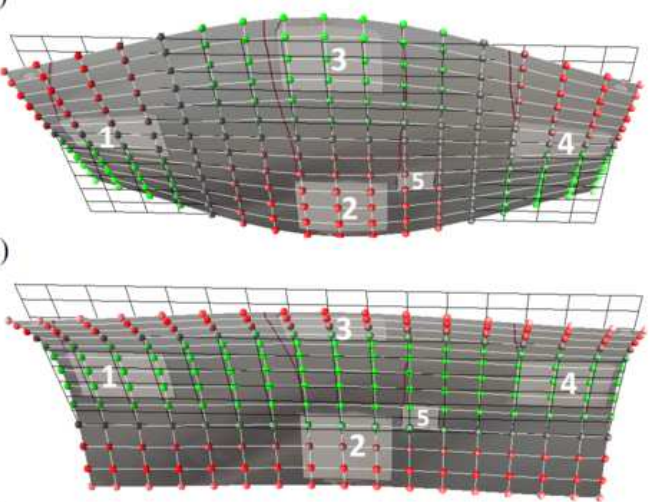

(e)

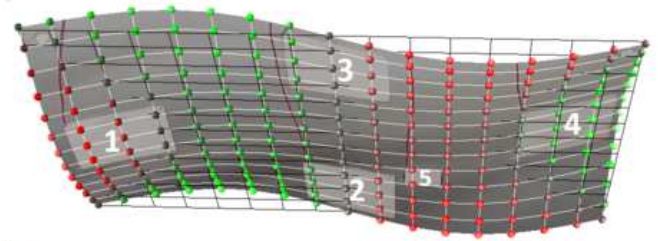

(f)

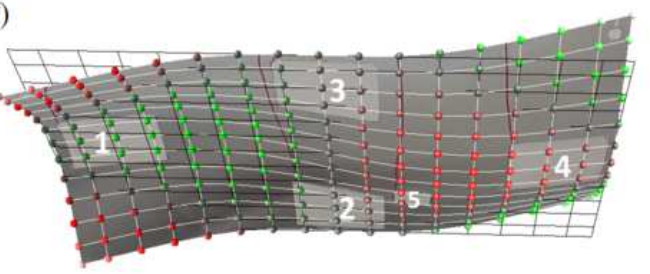

Figure 12. Resonant vibration modes 1-6(a-f) of free plate with five pairs of SC piezoceramic patches.

in Table 11 along with the mean deviations relative to the optimum inductance tuning in Table 9 . The comparison in Table 11 shows that the static capacitance $\left(C_{p}^{0}\right)_{i}$ is most accurate for the lower modes, while its deviation increases with frequency, confirming the observations from the previous free beam experiments. The opposite tendency is found for the tuning based on the blocked capacitance $C_{p}^{S}$, which becomes increasingly precise for higher target modes.

The influence on the damping performance, when using the inductance based on the static capacitance, is finally analyzed by considering the FRF for the plate corner displacements around target mode 6 shown in Figure 15(a) with the corresponding absorber voltage response in Figure 15(b). The exactly implemented inductors used in the experiment have mean inductance values around 
TABLE 9. Experimental SC and OC frequencies of free plate with 5 piezoceramic patch pairs and the effective EMCC, modal capacitances and optimum shunt tuning based on the FRFs for charge and voltage in Figure 11.

\begin{tabular}{lccccc}
\hline \multicolumn{7}{c}{ Experimental results } \\
Mode & 2 & 3 & 4 & 5 & 6 \\
\hline$\omega /(2 \pi)[\mathrm{Hz}]$ & 153.4 & 332.3 & 371.9 & 413.5 & 501.2 \\
$\hat{\omega} /(2 \pi)[\mathrm{Hz}]$ & 153.7 & 332.9 & 372.7 & 413.6 & 502.4 \\
$\kappa_{r}^{2}[\%]$ & 0.51 & 0.35 & 0.47 & 0.48 & 0.47 \\
\hline$\left(C_{r}\right)_{1}[\mathrm{nF}]$ & 243.7 & 250.4 & 239.8 & 252.5 & 223.6 \\
$\left(C_{r}\right)_{2}[\mathrm{nF}]$ & 235.8 & 230.6 & 235.0 & 240.7 & 244.5 \\
$\left(C_{r}\right)_{3}[\mathrm{nF}]$ & 225.7 & 217.9 & 223.4 & 213.7 & 216.1 \\
$\left(C_{r}\right)_{4}[\mathrm{nF}]$ & 246.9 & 259.3 & 243.2 & 245.1 & 222.3 \\
\hline$\left(L_{r}\right)_{1}[\mathrm{H}]$ & 4.38 & 0.910 & 0.756 & 0.586 & 0.447 \\
$\left(L_{r}\right)_{2}[\mathrm{H}]$ & 4.52 & 0.986 & 0.773 & 0.613 & 0.408 \\
$\left(L_{r}\right)_{3}[\mathrm{H}]$ & 4.72 & 1.040 & 0.812 & 0.693 & 0.462 \\
$\left(L_{r}\right)_{4}[\mathrm{H}]$ & 4.32 & 0.846 & 0.746 & 0.604 & 0.450 \\
\hline$\left(R_{r}\right)_{1}[\Omega]$ & 426 & 159 & 171 & 149 & 137 \\
$\left(R_{r}\right)_{2}[\Omega]$ & 441 & 173 & 175 & 156 & 125 \\
$\left(R_{r}\right)_{3}[\Omega]$ & 461 & 183 & 184 & 176 & 141 \\
$\left(R_{r}\right)_{4}[\Omega]$ & 421 & 151 & 169 & 153 & 138 \\
\hline
\end{tabular}

TABLE 10. The passive inductances and relative standard deviations (Std) and resistance values used in the experiment and the relative deviation to the optimum shunt tuning in Table 9.

\begin{tabular}{lcccc}
\hline Mode & 2 & 3 & 4 & 6 \\
\hline$\left(L_{r}\right)_{1}[\mathrm{H}]$ & - & - & 0.760 & 0.444 \\
Std [\%] & - & - & $(0.62)$ & $(0.62)$ \\
Deviation & - & - & $0.48 \%$ & $-0.64 \%$ \\
$\left(L_{r}\right)_{2}[\mathrm{H}]$ & 4.53 & 0.986 & 0.790 & - \\
Std [\%] & $(0.41)$ & $(0.54)$ & $(0.53)$ & - \\
Deviation & $0.29 \%$ & $0.06 \%$ & $2.30 \%$ & - \\
$\left(L_{r}\right)_{3}[\mathrm{H}]$ & 4.70 & 1.05 & 0.811 & - \\
Std $[\%]$ & $(0.53)$ & $(0.44)$ & $(0.66)$ & - \\
Deviation & $-0.47 \%$ & $0.41 \%$ & $-0.19 \%$ & - \\
$\left(L_{r}\right)_{4}[\mathrm{H}]$ & - & - & 0.751 & 0.446 \\
Std [\%] & - & - & $(0.57)$ & $(0.44)$ \\
Deviation & - & - & $0.60 \%$ & $-0.77 \%$ \\
\hline$\left(R_{r}\right)_{1}[\Omega]$ & - & - & 100 & 80 \\
Deviation & - & - & $-42 \%$ & $-41 \%$ \\
$\left(R_{r}\right)_{2}[\Omega]$ & 250 & 100 & 100 & - \\
Deviation & $-43 \%$ & $-42 \%$ & $-43 \%$ & - \\
$\left(R_{r}\right)_{3}[\Omega]$ & 250 & 100 & 100 & - \\
Deviation & $-46 \%$ & $-45 \%$ & $-46 \%$ & - \\
$\left(R_{r}\right)_{4}[\Omega]$ & - & - & 100 & 80 \\
Deviation & - & - & $-41 \%$ & $-42 \%$ \\
\hline
\end{tabular}

the target mode 6 given by $\left(L_{r}^{0}\right)_{1}=0.399 \mathrm{H}$ and $\left(L_{r}^{0}\right)_{4}=0.406 \mathrm{H}$, thus slightly larger than the tuning values in Table 11. It is clearly seen in Figure 15 that the piezoelectric absorbers are severely detuned when the inductances are based on the static capacitances (red dashed curves) compared 

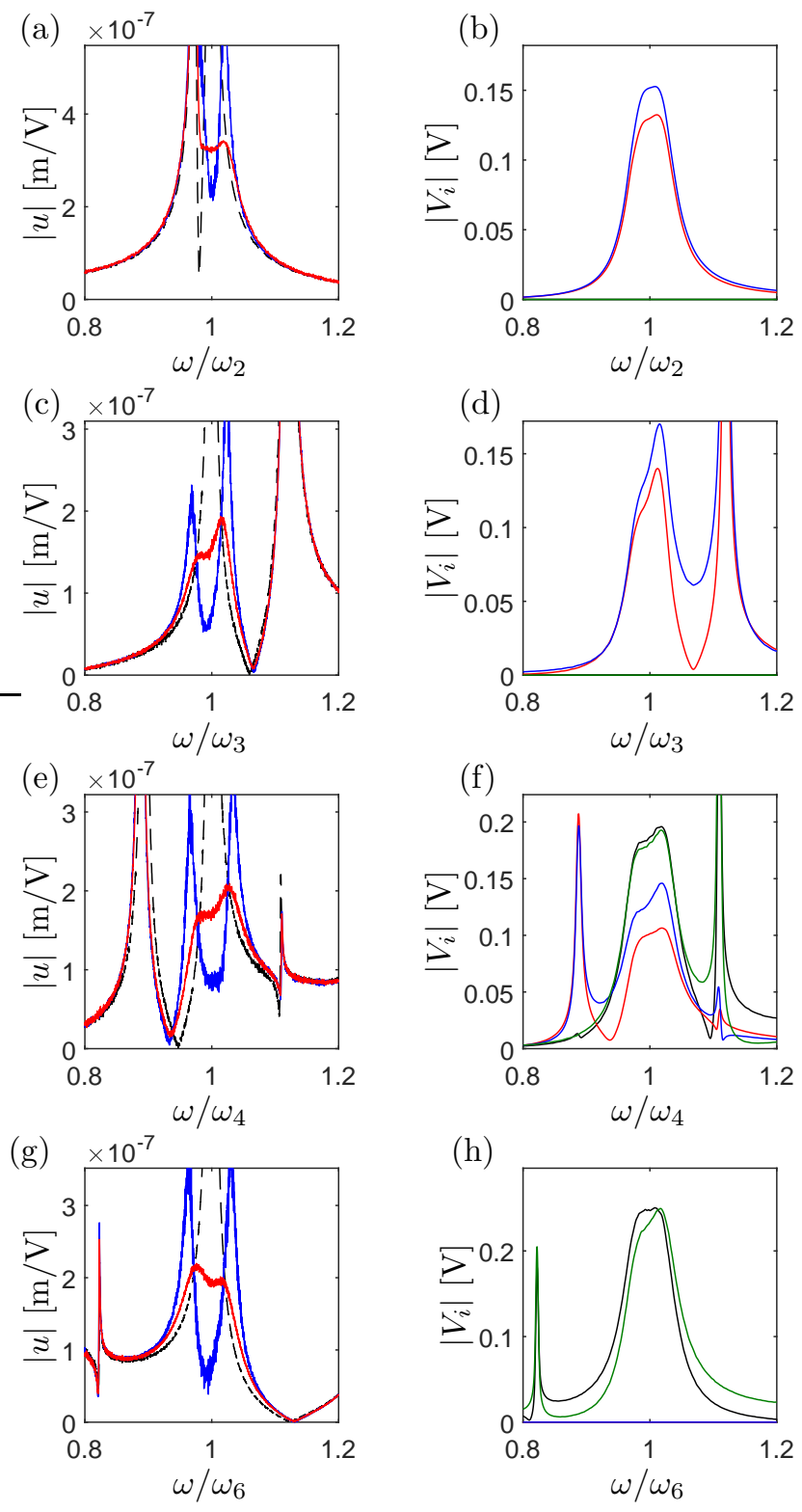

FiguRE 13. Experimental FRF for the corner displacements and voltages around mode $2(\mathrm{a}, \mathrm{b}), 3(\mathrm{c}, \mathrm{d}), 4(\mathrm{e}, \mathrm{f})$ and $5(\mathrm{~g}, \mathrm{~h})$ of free plate with 4 piezoelectric absorbers. In $(\mathrm{a}, \mathrm{c}, \mathrm{e}, \mathrm{g})$ the black, blue and red curves is the FRFs corresponding to the OC, and $L$ - and $L R$-shunted absorbers. In (b,d,f,h) the black, red, blue and green curves indicates the voltage response for absorber 1 to 4 .

to the response with optimally tuned absorbers (red solid curves) based on the effective modal capacitances. This demonstrates the importance of considering the residual mode contributions by the shunt tuning contained in the effective modal capacitances $\left(C_{r}\right)_{i}$ obtained by (11).

4.2.4. Multi-mode damping. Simultaneous damping of several vibration modes is now considered by calibrating the piezoelectric absorbers to different target modes. Initially, simultaneous damping of the closely spaced vibration modes 3 and 4 is considered by tuning the absorbers 2 and 3 to mode 3 , while absorbers 1 and 4 target mode 4 . Subsequently, the four piezoelectric absorbers 1 

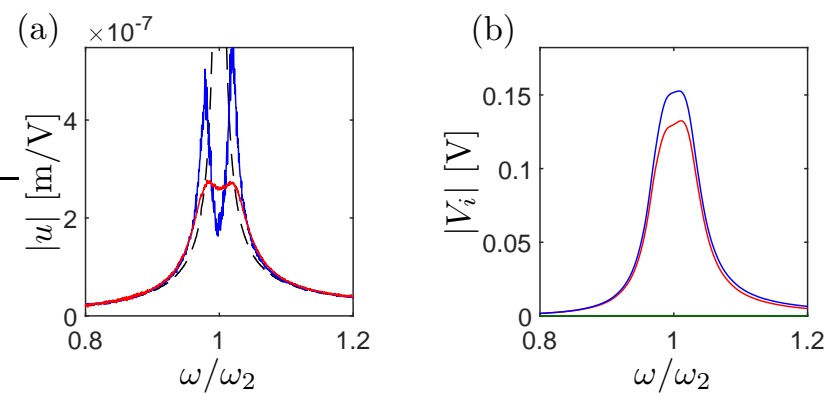

FiguRE 14. Experimental FRF for displacements of the center point on the left plate edge and voltages around mode 2 of free plate with 4 piezoelectric absorbers. In (a) the black, blue and red curves is the FRFs corresponding to the OC, and $L$ - and $L R$-shunted absorbers and in (b) the black, red, blue and green curves indicates the voltage response for absorber 1 to 4 .

TABLE 11. Shunt inductance tuning based on the experimental static $\left(C_{p}^{0}\right)_{i}$ and blocked $\bar{C}_{p}^{S}$ capacitances in Table 8 and the mean relative deviation (Dev) to the optimum tuning with residual mode contribution in Table 9.

\begin{tabular}{lccccc}
\hline Flex. mode & 2 & 3 & 4 & 5 & 6 \\
\hline$\left(L_{r}^{0}\right)_{1}[\mathrm{H}]$ & 4.19 & 0.893 & 0.713 & 0.581 & 0.392 \\
$\left(L_{r}^{0}\right)_{2}[\mathrm{H}]$ & 4.35 & 0.926 & 0.740 & 0.603 & 0.407 \\
$\left(L_{r}^{0}\right)_{3}[\mathrm{H}]$ & 4.57 & 0.974 & 0.777 & 0.634 & 0.428 \\
$\left(L_{r}^{0}\right)_{4}[\mathrm{H}]$ & 4.23 & 0.901 & 0.719 & 0.587 & 0.396 \\
Mean Dev. [\%] & -3.3 & -2.8 & -4.4 & -3.4 & -7.9 \\
\hline$\left(L_{r}^{S}\right)_{i}[\mathrm{H}]$ & 5.78 & 1.23 & 0.981 & 0.800 & 0.540 \\
Mean Dev. [\%] & 29 & 29 & 27 & 28 & 23 \\
\hline
\end{tabular}
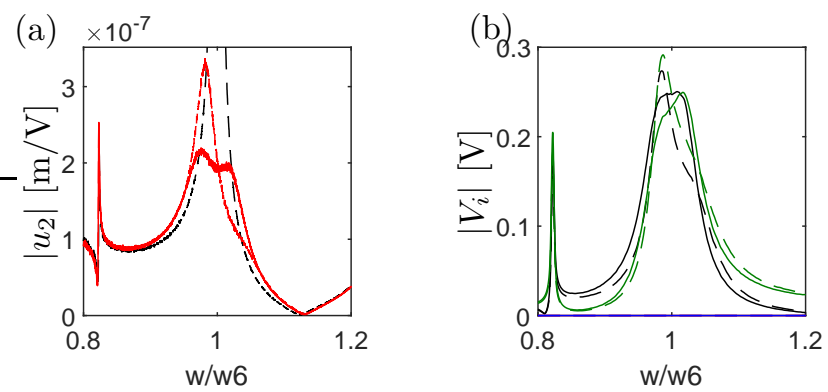

FiguRE 15. Comparison of experimental FRF for the optimum shunt tuning based on the effective (solid curves) and static (dashed curves) capacitances, (a) for plate corner displacements and (b) voltages of the piezoelectric absorbers 1 to 4.

to 4 are respectively tuned to modes $6,3,2$ and 4, for simultaneous damping of all four vibration modes.

As discussed in the section Numerical shunt tuning the optimum multi-mode shunt tuning is based on the same charge and voltage FRF curves, by evaluating the modified effective EMCC $\tilde{\kappa}_{r}^{2}$ in (13), which only considers the coupling of the piezoelectric absorber(s) tuned to the specific targeted mode. Therefore, the multi-mode shunt tuning of the piezoelectric absorbers is based on the FRFs for measured charge and voltage in Figure 11, also have previously been used for single mode tuning. Table 12 provides the modified effective EMCC, the corresponding $L R$-shunt tuning 
TABLE 12. Modified effective EMCC and corresponding series shunt tuning of the plate bonded piezoelectric absorbers 1 to 4 in Figure 6(c). (...): Implemented resistances.

\begin{tabular}{lcccc}
\hline \multicolumn{5}{c}{ Multi-mode tuning } \\
Mode & 3 and 4 & Dev. & $2,3,4$ and 6 & Dev. \\
\hline$\tilde{\kappa}_{2}^{2}[\%]$ & - & & 0.23 & $-51 \%$ \\
$\tilde{\kappa}_{3}^{2}[\%]$ & 0.35 & $-0.1 \%$ & 0.16 & $-53 \%$ \\
$\tilde{\kappa}_{4}^{2}[\%]$ & 0.34 & $-27 \%$ & 0.27 & $-47 \%$ \\
$\tilde{\kappa}_{6}^{2}[\%]$ & - & & 0.18 & $-62 \%$ \\
\hline$\left(L_{r}\right)_{1}[\mathrm{H}]$ & 0.758 & $0.1 \%$ & 0.448 & $0.2 \%$ \\
$\left(L_{r}\right)_{2}[\mathrm{H}]$ & 0.988 & $0.0 \%$ & 0.987 & $0.2 \%$ \\
$\left(L_{r}\right)_{3}[\mathrm{H}]$ & 1.043 & $0.0 \%$ & 4.735 & $0.2 \%$ \\
$\left(L_{r}\right)_{4}[\mathrm{H}]$ & 0.747 & $0.1 \%$ & 0.748 & $0.2 \%$ \\
\hline$\left(R_{r}\right)_{1}[\Omega]$ & $147(70)$ & $-16 \%$ & $96(50)$ & $-30 \%$ \\
$\left(R_{r}\right)_{2}[\Omega]$ & $172(100)$ & $0.0 \%$ & $119(50)$ & $-31 \%$ \\
$\left(R_{r}\right)_{3}[\Omega]$ & $183(100)$ & $0.0 \%$ & $337(125)$ & $-27 \%$ \\
$\left(R_{r}\right)_{4}[\Omega]$ & $144(70)$ & $-16 \%$ & $105(40)$ & $-38 \%$ \\
\hline
\end{tabular}

and its deviation from the basic single-mode tuning for simultaneous damping of modes 3 and 4 and simultaneous damping of modes $2,3,4$ and 6 .

For the simultaneous damping of modes 3 and 4 it is seen that the modified effective EMCC for mode 3 in Table 12 is equal to the original effective EMCC, which is the case because the piezoelectric absorbers 1 and 4 have limited coupling with this specific mode. The remaining effective EMCCs are as expected seen to be somewhat lower than the original effective EMCC, as fewer piezoelectric absorbers now contribute to the overall coupling of a targeted mode. For all cases it can be seen in Table 12 that the influence from the modified effective EMCC on the inductance tuning is negligible, whereby the same passive inductors from the single mode tuning can in fact be implemented for multi-mode damping. The optimum resistances are however seen to be significantly altered, since their values are proportional to the square root of the modified effective EMCC, see Table 1. The new resistances used for the experimental multi-mode shunt tuning are thus in Table 12 provided in parentheses for comparison. These implemented values are as well smaller than the optimum tuning, mainly because of the inherent resistance in the passive inductors. However, the deviation between the optimal and the implemented resistances are seen to have approximately the same magnitude as for the single mode tuning, which indicates that the modified effective EMCC significantly alters the required resistance.

The FRF for the plate corner displacements and the corresponding absorber voltage response can be seen in Figure 16 for the simultaneous damping of modes 3 and 4 and in Figure 17 for the damping of modes 2, 3, 4 and 6 . It can be seen from the FRF for the plate corner displacements in Figure 16(a) that both modes 3 and 4 are optimally damped by the $L R$-shunted absorbers (red curves) with flat plateaus around the two target modes. Furthermore, it can be seen in Figure 16(b) that there is almost no absorber voltage response for absorber 1 (black) and 4(green) around mode 3 and for absorber 2(red) and 3(blue) around mode 4. This limited spill-over to non-targeted modes indicates precise tuning of the individual absorbers to their respective target modes.

Similar characteristics can be seen for the simultaneous damping of modes 2,3 , 4, and 6 in Figure 17, in which flat plateaus around the target modes can be observed in the FRF for the plate corner displacements in Figure 17(a), except for the first resonance peak of the undamped first free plate mode in Figure 12(a). Furthermore, it is clearly seen from the FRF for the absorber voltage in Figure 17(b), that significant voltage amplitudes are present only around the respective target modes of the individual piezoelectric absorbers. 

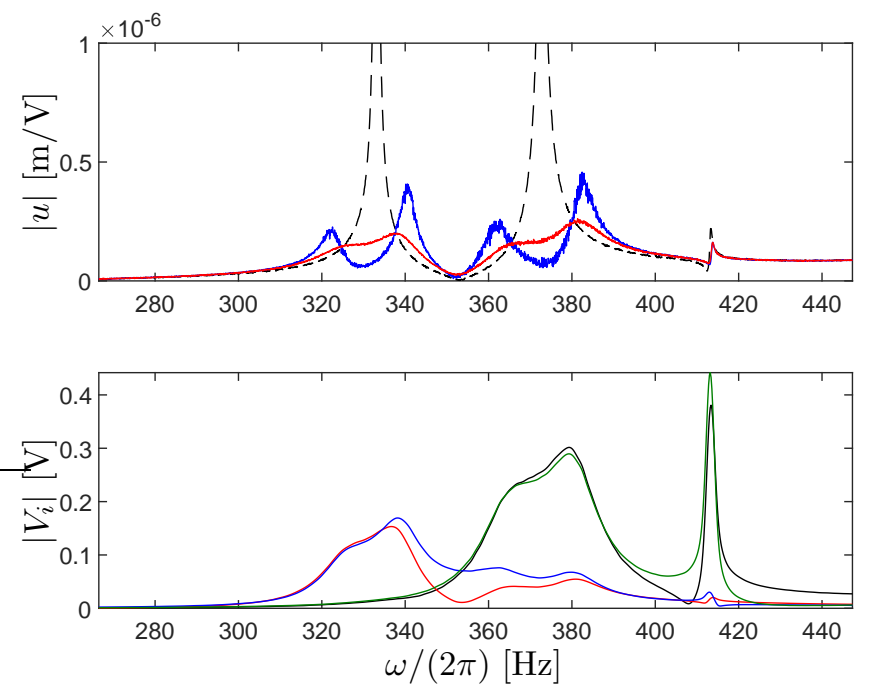

FiguRE 16. Experimental FRF for (a) the plate corner displacements with OC(dashed), $L$ (blue)- and $L R$ (red)-shunted absorbers and the voltage response (b) of the piezoelectric absorbers 2(red) and 3(blue) tuned to mode 3, and absorber 1(black) and 4(green) tuned to mode 6 .
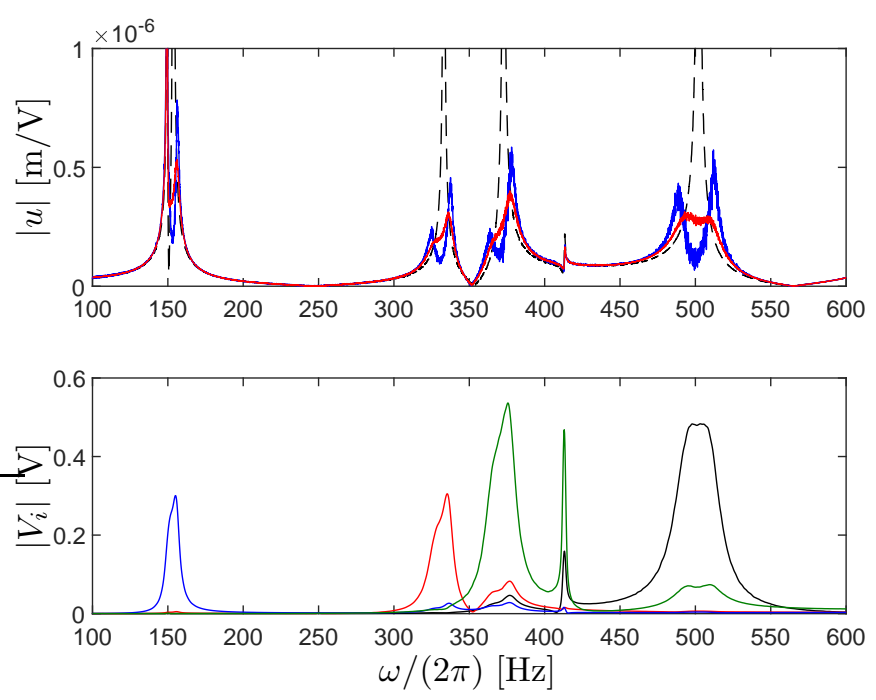

FiguRE 17. Experimental FRF for (a) the plate corner displacements with OC(dashed), $L$ (blue)- and $L R$ (red)-shunted absorbers and the voltage response (b) of the piezoelectric absorbers 1(black), 2(red), 3(blue) and 4(green) tuned to mode $6,3,2$ and 4 , respectively.

\section{CONCLUSION}

The use of passive inductors for resonant piezoelectric shunt tuning requires a precise prior inductance tuning which accounts for the influence from the residual vibration modes. This is done in a recently proposed shunt tuning method by the evaluation of an effective modal capacitance determined from a SC modal charge and OC modal voltage obtained by the evaluation of the two corresponding eigenvalue problems. In the present paper, it is demonstrated how this method can be based on simple measurements of the piezoelectric absorber SC current and OC voltage response to a pseudo-random vibration excitation of the electromechanical structure. By 
acquiring the current and voltage measurements with a suitable sample interval and time-span the corresponding FRFs may be obtained with high accuracy. From the FRF for the electric current the corresponding FRF for electric charge is obtained and the shunt tuning is then entirely based on the FRFs for the absorber charge and voltage responses. The modal charge and voltage are then found as the peak amplitudes in the FRFs, while SC and OC frequencies follows by the intersection with the frequency axis. The effective modal capacitance is finally evaluated from the modal charge and voltage and used for the optimum shunt tuning instead of the traditional use of the blocked or static capacitances.

The experimental shunt tuning method is demonstrated for, respectively, a free beam and plate with five pairs of piezoceramic patches. In both cases one piezoceramic patch pair is used to excite structural vibrations, while the remaining four pairs are connected in series with a resonant shunt, whereby they act as four individual piezoelectric absorbers. For the free beam the respective tuning and damping of four resonant vibration modes is considered and shown to be in good agreement with the corresponding numerical results obtained with 3D FEs in ANSYS. For the free plate optimum multi-mode shunt damping is demonstrated by tuning the piezoelectric absorbers to different target modes, while altering the resistance tuning by a modified effective EMCC. Finally, it is shown that tuning of the shunt inductance based on the blocked capacitance for the 2nd beam mode and the static capacitance for the 6th plate mode causes severe detuning of the piezoelectric shunts and reduces the attainable damping significantly. It can thus be concluded that a precise inductance tuning is vital for the shunt damping performance, especially for pure passive resonant shunts where the re-adjustments of the shunt inductance is not straight forward. A precise prior tuning is therefore required and may only be ensured by taking into account the contribution from the residual vibration modes.

\section{FUNDING}

This research has been supported by the Danish Council for Independent Research via the project 'Resonant Piezoelectric Shunt Damping of Structures'.

\section{REFERENCES}

[1] Berardengo M, Thomas O, Giraud-Audine C and Manzoni S (2016) Improved resistive shunt by means of negative capacitance: new circuit, performances and multi-mode control. Smart Mater. and Struct. 25:075033(23pp).

[2] Berardengo M, Manzoni S, Thomas O, and Vanali M (2018) Piezoelectric resonant shunt enhancement by negative capacitances: Optimisation, performance and resonance cancellation. J. Intell. Mater. Syst. Struct. 29(12):2581-2606.

[3] Caruso G (2001) A critical analysis of electric shunt circuits employed in piezoelectric passive vibration damping. Smart Mater. Struct. 10:1059-68.

[4] CTS (2019) List of piezoelectric materials PZT 3265HD. https://www.ctscorp.com/wp-content/uploads /CTS_-PZT-Materials_Complete-Properties _20180829.pdf

[5] Fleming A J, Behrens S and Moheimani S O R (2000) Synthetic impedance for implementation of piezoelectric shunt-damping circuits. Electronics Letters, 36:1525-6.

[6] Forward R L (1979) Electronic damping of vibrations in optical structures. Appl. Optics. 18:690-7.

[7] Gardonio P and Casagrande D (2017) Shunted piezoelectric patch vibration absorber on two-dimensional thin structure: tuning considerations. J. Sound Vib. 395:26-47.

[8] Hagood N W and von Flotow A (1991) Damping of structural vibrations with piezoelectric materials and passive electrical networks. J. Sound Vib. 146:243-68.

[9] Høgsberg J and Krenk S (2012) Balanced calibration of resonant shunt circuits for piezoelectric vibration control. J. Intell. Mater. Syst. Struct. 23:1937-48.

[10] Høgsberg J and Krenk S (2017) Calibration of piezoelectric RL shunts with explicit residual mode correction. J. Sound Vib. 386:65-81. 
[11] IEEE Inc. (1988) Standards on piezoelectricity ANS/IEEE Std 176-1987 USA.

[12] Krenk S and Høgsberg J (2016) Tuned resonant mass or inerter-based absorbers: Unified calibration with quasi-dynamic flexibility and inertia correction. Proc. R. Soc. A. 472:20150718(23pp).

[13] Krenk S and Høgsberg J (2013) Equal modal damping design for a family of resonant vibration control formats. J. Vib. Cont. 19:1294-315.

[14] Lossouarn B, Aucejo M, Deü J F and Multon B (2017) Design of inductors with high inductance values for resonant piezoelectric damping. Sens. Act. A: Phys. 259:68-76.

[15] de Marneffe B and Preumont A (2008) Vibration damping with negative capacitance shunts: theory and experiment. Smart Mater. Struct. 17:035015.

[16] Park C H and Inman D J (1999) A uniform model for series R-L and parallel R-L shunt circuits and power consumption. SPIE Proceedings 3668:797-804.

[17] Porfiri M, Maurini C and Pouget J (2007) Identification of electromechanical modal parameters of linear piezoelectric structures. Smart Mater. Struct. 16:323-31.

[18] Preumont A (2011) Vibration Control of Active Structures. An Introduction 3rd edition Springer Heidelberg.

[19] Soltani P, Kerschen G, Tondreau G and Deraemaeker A (2014) Piezoelectric vibration damping using resonant shunt circuits: an exact solution. Smart Mater. Struct. 23:125014(11pp).

[20] Thomas O, Ducarne J and Deü J F (2012) Performance of piezoelectric shunts for vibration reduction. Smart Mater. Struct. 21:015008(16pp).

[21] Toftekær J F, Benjeddou A, Høgsberg J and Krenk S (2018) Optimal piezoelectric RL shunt damping of plates with residual mode correction. J. Intell. Mater. Syst. Struct. 29:3346-70.

[22] Toftekær J F, Benjeddou A and Høgsberg J (2019) General numerical implementation of a new piezoelectric shunt tuning method based on the effective electromechanical coupling coefficient. Mech. Adv. Mater. Struc.

[23] Toftekær J F and Høgsberg J (2019) Multi-Mode Piezoelectric Shunt Damping with Residual Mode Correction by Evaluation of Modal Charge and Voltage. J. Intell. Mater. Syst. Struct. (submitted).

[24] Wu S Y (1996) Piezoelectric shunts with a parallel R-L circuit for structural damping and vibration control. In Proc. SPIE 2720:259-69.

[25] Wu S Y and Bicos A S (1997) Structural vibration damping experiments using improved piezoelectric shunts. In Proc. SPIE 3045:40-50.

[26] Yamada K, Matsuhisa H, Utsuno H and Sawada K (2010) Optimum tuning of series and parallel LR circuits for passive vibration suppression using piezoelectric elements. J. Sound Vib., 329:5036-57. 